\title{
A Network Pharmacology Study on the Active Components and Targets of the Radix Ginseng and Radix Bupleuri Herb Pair for Treating Nonalcoholic Fatty Liver Disease
}

\author{
Qiang Zhang, ${ }^{1}$ Li Zhang, ${ }^{1}$ Kaili Liu, ${ }^{1}$ Haonan Shang, ${ }^{1}$ Jun Ruan, ${ }^{1}$ Zhonghai Yu $\left(\mathbb{D},{ }^{1}\right.$ \\ Shengxi Meng, ${ }^{1}$ Fang Liang, ${ }^{1}$ Tianzhan Wang, ${ }^{1}$ Hongyan Zhang, ${ }^{1}$ Wenbo Peng, \\ Yuxin Wang, ${ }^{1}$ Junming Chen, ${ }^{2}$ Tiegang Xiao ${ }^{(D)}{ }^{3}$ and Bing Wang ${ }^{1}$ \\ ${ }^{1}$ Department of Traditional Chinese Medicine, Shanghai Sixth People's Hospital Affiliated to Shanghai Jiao Tong University, \\ Shanghai, China \\ ${ }^{2}$ Department of Traditional Chinese Medicine, Shanghai Pudong New District Zhoupu Hospital, \\ Shanghai University of Medicine \& Health Sciences, Shanghai, China \\ ${ }^{3}$ Department of Gastroenterology, Shanghai Traditional Chinese Medicine-Integrated Hospital, \\ Shanghai University of Traditional Chinese Medicine, Shanghai, China
}

Correspondence should be addressed to Tiegang Xiao; xiaotiegang1@163.com and Bing Wang; bingliver@163.com

Received 14 May 2021; Accepted 21 December 2021; Published 8 February 2022

Academic Editor: Byung-Cheol Lee

Copyright ( 2022 Qiang Zhang et al. This is an open access article distributed under the Creative Commons Attribution License, which permits unrestricted use, distribution, and reproduction in any medium, provided the original work is properly cited.

Objective. To explore the potential active components and corresponding target herb pairs of Radix Ginseng (Renshen) and Radix Bupleuri (Chaihu) in the treatment of nonalcoholic fatty liver disease (NAFLD) through network pharmacology and in vitro experiments. Methods. The active components and potential targets of the herb pair of Renshen and Chaihu were screened through a network database system, and Venn analysis was performed with the obtained NAFLD targets. The intersecting targets were analysed for gene ontology (GO) functions and Kyoto Encyclopedia of Genes and Genome (KEGG) pathways, and a proteinprotein interaction (PPI) network was generated. Cytoscape software was used to construct active component-target networks of the Renshen and Chaihu herb pair. Free fatty acids were added to the HepG2 cell line to create high-fat models that were treated with different concentrations of stigmasterol. The effect of stigmasterol on the lipid metabolism in HepG2 cells and PPAR $\gamma$ knockdown cells was determined by oil red O staining, Nile red staining, and TG level. PPAR $\gamma$ and UCP-1 mRNA, and protein expression levels were detected by qRT-PCR and Western blot analyses, respectively. Results. Twenty active components obtained from the Renshen and Chaihu herb pair were identified. The herb pair active component-target network showed that both Renshen and Chaihu contained stigmasterol and kaempferol as active components. The PPI network comprised 63 protein nodes. GO enrichment analysis and KEGG pathway enrichment analysis showed that the targets were mainly involved in lipid metabolism. Eight core targets were identified: AKT1, PPARG, MAPK3, TNF, TP53, SIRT1, STAT3, and PPARA. In vitro experiments demonstrated that stigmasterol reduced lipid accumulation and TG levels in HepG2 cells, and the mechanism may have been related to the activation of the PPAR $\gamma$-UCP-1 signalling pathway. Conclusion. This study preliminarily illustrated the potential components and corresponding core targets of the Renshen and Chaihu herb pair in treating NAFLD. The effect of stigmasterol on the PPAR $\gamma$-UCP-1 signalling pathway in enhancing lipid metabolism may represent one of the mechanisms of the Renshen and Chaihu herb pair in the treatment of NAFLD. The results provide new evidence and research insights to reveal the roles of Renshen and Chaihu in the management of NAFLD. 


\section{Introduction}

Nonalcoholic fatty liver disease (NAFLD) is caused by metabolic stress-induced liver injury closely related to insulin resistance and genetic susceptibility. One of the most common hepatic diseases worldwide, NAFLD is a spectrum disease that includes nonalcoholic fatty liver (NAFL), nonalcoholic steatohepatitis (NASH), and liver cirrhosis [1]. A recent systematic review has indicated that the prevalence of NAFLD is as high as $29.2 \%$ in China [2]. The pathophysiology of NAFLD is not yet clear; however, lipotoxic response is clearly a central mechanism of NAFLD development and progression [3]. Based on this understanding, various chemical agents have been developed and are under investigation to explore their potential benefits [4]. However, no drugs specific for NAFLD have been approved to date $[5,6]$. In China, many patients seek medical consultations from practitioners of traditional Chinese medicine (TCM) and consume Chinese herbal medicines (CHMs).

Various studies have found the potential therapeutic effect of CHMs on NAFLD [7-9]. The Radix Ginseng (Renshen) and Radix Bupleuri (Chaihu) herb pair is a commonly prescribed herb combination. Nevertheless, the specific active components are unknown. In addition, due to the complexity of active components in Renshen and Chaihu, other underlying mechanisms may confer protection against NAFLD. These issues deserve further investigation.

Network pharmacology, characterized by the comprehensive presentation of herb-target-disease networks, may be a valuable approach to explore the outstanding issues regarding the herb pair effect on NAFLD $[10,11]$. Network pharmacology can be used to identify the potential active components and corresponding therapeutic targets of the Renshen and Chaihu herb pair for treating NAFLD. Additional precise identifications can then be made to verify their effects.

In this study, we first used the network database to identify the active components of herbs similar to the Renshen and Chaihu herb pair and employed SwissTargetPrediction to predict the potential therapeutic targets. Then, an intersection analysis of potential targets of the herb pair and known therapeutic targets of NAFLD was performed to identify the core targets. In addition, a PPI analysis, GO enrichment analysis, and KEGG pathway enrichment analysis were performed, and an active component-target network was established. Finally, in vitro experiments verified that the Renshen and Chaihu herb pair had a pharmacodynamic effect on the components of stigmasterol by activating the PPAR $\gamma$ signalling pathway, clarified the material basis of the Renshen and Chaihu herb pair for the treatment of NAFLD, and provided an experimental basis for the treatment of NAFLD.

\section{Methods}

2.1. Data Preparation. A total of 39 active components in the herb pair were manually extracted from the TCM System Pharmacology Database (TCMSP, http://sp.nwu.edu.cn/ tcmsp.php, version 2.3) [12]. Relationships among drug components, targets, and diseases were obtained from the
TCMSP; this database contains the pharmacokinetic properties of a variety of Chinese medicines, including their oral bioavailability, similar herbs, and intestinal epithelial permeability.

2.2. Active Component Prediction. After active component screening, an absorption, distribution, metabolism, and excretion (ADME) evaluation system was used to select potential active components. We selected two pharmacokinetic parameters, namely, oral bioavailability (OB) [13] and herb similarity (DL) [14], to identify the active components of the herb pair. In this study, $\mathrm{OB} \geq 30 \%$ and $\mathrm{DL} \geq 0.18$ were used as the screening criteria for active components. Active components that met the criteria were regarded as candidate components for subsequent analysis.

2.3. Target Fishing. To obtain the $2 \mathrm{D}$ structure of active components, we evaluated chemical data in PubChem (https://pubchem.ncbi.nlm.nih.gov) and saved the 2D structure in sdf format. PubChem is the largest freely accessible database of chemical information in the world, providing chemical properties, physical properties, biological activity, safety, and toxicity information of chemical substances. The 2D structure of the active components was sequentially imported into SwissTargetPrediction (http:// www.swisstargetprediction.ch) [15], which was used to predict the most likely macromolecular target based on the $2 \mathrm{D}$ or $3 \mathrm{D}$ structure of the biologically active small molecules. We selected Homo sapiens as the species to predict the potential targets of Renshen and Chaihu.

2.4. The Disease Target of NAFLD. NAFLD-related genes were obtained from the DisGeNET database (https://www. disgenet.org) [16]. The DisGeNET database has publicly available genes and variants related to human diseases. We used the key term "non-alcoholic fatty liver disease" to search, and we obtained 333 genes. We then performed a Venn diagram analysis of the intersection of the active components and NAFLD genes.

2.5. Protein-Protein Interaction Data. The PPI data of 63 intersection targets of the herb pair and NAFLD were extracted from STRING (http://string-db.org/) [17], and the parameters were filtered using Homo sapiens (confidence level >0.4). The STRING database is frequently used to generate PPI networks and mine core regulatory genes.

2.6. GO Enrichment Analysis and KEGG Pathway Enrichment Analysis. The intersection target of NAFLD and the herb pair was analysed through gene ontology (GO) analysis and Kyoto Encyclopedia of Genes and Genomes (KEGG) pathway analysis in the Metascape database (https:// metascape.org/) [18]. An adjusted $P$ value $<0.05$ was set as the standard, and the species was set to Homo sapiens. The biological processes and pathways related to NAFLD were obtained. 
2.7. Topological Analysis of the Active Component-Target Network. Topological analysis was performed by importing TSV format files from STRING to Cytoscape 3.7.02 software. Cytoscape was used to visualize biological pathway networks and molecular interaction networks. First, we calculated the topology parameters of the PPI network through the NetworkAnalyser tool. Then, using the degree parameter, we sorted the target area from large to small and the colour from light to dark. Using the Generate Style in the Statistics tool, we sorted the lines between the targets from thick to thin according to the combined score, and we sorted the colours from light to dark. On the basis of results greater than the median of the twofold degree value of the PPI network, we identified the core targets for the regulation of NAFLD by active components. Finally, we obtained 8 core targets and matched them with the active components. Thus, we identified 20 active components related to the 8 core targets from the intersection targets.

2.8. Network Construction. An active component-target network was established. The core target and its matching active components were obtained on the strength of PPI data and topological analysis. Then, the network of herb pair active component-core target of NAFLD was constructed.

\subsection{Establishment of the NAFLD Cell Model. The AML12} normal mouse liver cell line and HepG2 human liver cancer cell line were donated by the Institute of Digestive Diseases of Shanghai University of Traditional Chinese Medicine. Both cell lines were seeded in 6-well plates and subcultured in the laboratory in DMEM containing 10\% foetal bovine serum and two antibiotics at $37^{\circ} \mathrm{C}$ in $95 \%$ air humidity and $5 \% \mathrm{CO}_{2}$ and maintained in good condition. Passaged cells were digested with $0.25 \%$ trypsin.

2.10. Experiment Grouping and Modelling. When reaching $60 \%-70 \%$ confluency, the cells were randomly divided into a blank control group (C), model group (M), low-dose stigmasterol group (L), and high-dose stigmasterol group $(\mathrm{H})$. Group M was supplemented with $400 \mu \mathrm{M}$ oleic acid and $200 \mu \mathrm{M}$ palmitic acid. In addition to free fatty acids, the $\mathrm{L}$ and $\mathrm{H}$ groups were stimulated with low-dose $(30 \mu \mathrm{M})$ and high-dose $(60 \mu \mathrm{M})$ stigmasterol, respectively. The cells were treated for 24 hours, and each group was set up with 3 replicate wells. The cells were incubated in an incubator at $37^{\circ} \mathrm{C}$ with $5 \% \mathrm{CO}_{2}$ and saturating humidity.

2.11. Efficacy Testing. Oil red $\mathrm{O}$ staining and Nile red staining were used to observe cell changes and the number of lipid droplets under a microscope to determine the pathological changes of the cells. A kit was used to detect biochemical indicators, including TG levels. RT-PCR was performed to detect the mRNA expression levels of PPAR $\gamma$, $\operatorname{PPAR} \alpha, \mathrm{CPT}-2$, and UCP-1. Western blot analysis was performed to detect the protein expression levels of PPAR $\gamma$, $\operatorname{PPAR} \alpha, \mathrm{CPT}-2$, and UCP-1. Immunofluorescence technology was used to detect PPAR $\gamma$ and $\operatorname{PPAR} \alpha$.
2.12. Generation of PPAR $\gamma$-Knockdown Cell Lines. CRISPR/Cas9 technology was used to construct PPAR $\gamma$ knockdown cell lines. Resuspended 293T cells were seeded in a culture dish. Transfection reagent $(30 \mu \mathrm{l})$ and Opti-MEM $(100 \mu \mathrm{l})$ medium were then mixed in a $1.5 \mathrm{EP}$ tube and incubated at room temperature for 5 minutes. PMG2D, PSPAX2, and the target plasmid were mixed with $100 \mu \mathrm{l}$ of the Opti-MEM medium in a $1.5 \mathrm{EP}$ tube at a ratio of $1: 3: 4$ (total of $2.5 \mu \mathrm{g}$ ) and incubated at room temperature for 5 minutes. The solutions were then mixed together and incubated at room temperature for 20 minutes. The medium $(10 \mathrm{ml})$ was added to the culture plate followed by the addition of the mixture, and the medium was changed after 24 hours of cultivation. The virus was harvested after 48 hours of cultivation. The original medium was removed from the HepG2 cells, and the virus was added to the culture dish. In addition, we added $1 \%$ polybrene to enhance the infection efficiency. After 24 hours of infection, the normal medium was changed, and the screening marker was added 48 hours later. After stable proliferation, a cell line expressing knocked down PPAR $\gamma$ was obtained.

2.13. Statistical Analysis. After performing a uniformity test of variance, the results were analysed by one-way analysis of variance (ANOVA) for comparison. $P<0.05$ was considered statistically significant.

\section{Results}

3.1. Network Database Analysis of the Effective Material Basis of the Renshen and Chaihu Herb Pair. According to the defined value of bioavailability, herb-like properties, and deduplication processing, 17 active components contained in Chaihu (Table 1) and 22 active components contained in Renshen (Table 2) were obtained from the TCMSP database. Targets of the active components were predicted. After removing duplications, 592 targets were obtained. Renshen and Chaihu herb pair active component-potential target regulatory networks were constructed using Cytoscape 3.7.2 software (Figure 1(a)). The 592 targets of the Renshen and Chaihu herb pair were crossed with the 333 NAFLD-related targets obtained from the DisGeNET database. In total, 63 crossed targets were screened, including HSD11B1, CNR2, PTPN1, CYP17A1, NR1H4, GPR119, FABP4, PPARD, APP, TEK, TNF, ALDH2, FDFT1, NPC1L1, NR1H3, SREBF2, CYP2C19, PPARA, VDR, PPARG, ALOX15, F2, ALOX5, GSK3B, MMP13, AKT1, INSR, TERT, ESRRA, MTOR, ITK, ADAM17, JAK2, CASP1, DPP4, REN, SIRT1, RBP4, GCK, MMP1, CPT1A, TRPV1, NAMPT, STAT3, FABP5, FABP1, MAPK3, PRKCE TP53, FFAR4, NR0B2 AGTR1, HNF4A, S1PR1, NQO1, SHH, NR1I2, CDK8, NR3C2, CCNC, TGFB1, PRKAB1, and PRKAA2 (Figure 1(b)).

The 63 overlapping target genes were subjected to GO enrichment analysis and KEGG pathway enrichment analysis. The GO enrichment analysis was performed to identify target genes involved in the biological process (BP), cellular component (CC), and molecular function (MF) categories. The top 8 terms were used to create a bar graph according to 
TABLE 1: Basic information on the 17 active components in Chaihu.

\begin{tabular}{|c|c|c|c|}
\hline Molecular ID & Name & $\mathrm{OB}$ & $\mathrm{DL}$ \\
\hline MOL001645 & Linoleyl acetate & 42.1 & 0.2 \\
\hline MOL002776 & Baicalin & 40.12 & 0.75 \\
\hline MOL000449 & Stigmasterol & 43.83 & 0.76 \\
\hline MOL000354 & Isorhamnetin & 49.6 & 0.31 \\
\hline MOL000422 & Kaempferol & 41.88 & 0.24 \\
\hline MOL004598 & 3,5,6,7-Tetramethoxy-2-(3,4,5-trimethoxyphenyl) chromone & 31.97 & 0.59 \\
\hline MOL004609 & Areapillin & 48.96 & 0.41 \\
\hline MOL013187 & Cubebin & 57.13 & 0.64 \\
\hline MOL004624 & Longikaurin A & 47.72 & 0.53 \\
\hline MOL004628 & Octalupine & 47.82 & 0.28 \\
\hline MOL004644 & Sainfuran & 79.91 & 0.23 \\
\hline MOL004648 & Troxerutin & 31.6 & 0.28 \\
\hline MOL004653 & (+)-Anomalin & 46.06 & 0.66 \\
\hline MOL004702 & Saikosaponin c_qt & 30.5 & 0.63 \\
\hline MOL004718 & $\alpha$-Spinasterol & 42.98 & 0.76 \\
\hline MOL000490 & Petunidin & 30.05 & 0.31 \\
\hline MOL000098 & Quercetin & 46.43 & 0.28 \\
\hline
\end{tabular}

TABLE 2: Basic information on the 22 active components in Renshen.

\begin{tabular}{lccc}
\hline Molecular ID & Name & OB & DL \\
\hline MOL002879 & Diop & 43.59 & 0.39 \\
MOL000449 & Stigmasterol & 43.83 & 0.76 \\
MOL000358 & -Sitosterol & 36.91 & 0.75 \\
MOL003648 & Inermin & 65.83 & 0.54 \\
MOL000422 & Kaempferol & 41.88 & 0.24 \\
MOL004492 & Chrysanthemaxanthin & 38.72 & 0.58 \\
MOL005308 & Aposiopolamine & 66.65 & 0.22 \\
MOL005314 & Celabenzine & 101.88 & 0.49 \\
MOL005317 & Deoxyharringtonine & 39.27 & 0.81 \\
MOL005318 & Dianthramine & 40.45 & 0.2 \\
MOL005320 & Arachidonate & 45.57 & 0.2 \\
MOL005321 & Frutinone A & 65.9 & 0.34 \\
MOL005344 & Ginsenoside rh2 & 36.32 & 0.56 \\
MOL005348 & Ginsenoside-Rh4_qt & 31.11 & 0.78 \\
MOL005356 & Girinimbin & 61.22 & 0.31 \\
MOL005357 & Gomisin B & 31.99 & 0.83 \\
MOL005360 & Malkangunin & 57.71 & 0.63 \\
MOL005376 & Panaxadiol & 33.09 & 0.79 \\
MOL005384 & Suchilactone & 57.52 & 0.56 \\
MOL005399 & Alexandrine_qt & 36.91 & 0.75 \\
MOL005401 & Ginsenoside Rg5_qt & 39.56 & 0.79 \\
MOL000787 & Fumarine & $\mathbf{5 9 . 2 6}$ & $\mathbf{0 . 8 3}$ \\
\hline
\end{tabular}

adjusted $P$ value rankings (Supplemental Table 1, Figure $1(\mathrm{c})$ ). The genes in the biological process category are involved in the regulation of small-molecule metabolic processes, regulation of lipid metabolic processes, positive regulation of small-molecule metabolic processes, positive regulation of lipid metabolic processes, steroid metabolic processes, lipid localization, transcription initiation from the RNA polymerase II promoter, and lipid transport. In the cellular component category, the genes are involved in the RNA polymerase II transcription factor complex, nuclear transcription factor complex, membrane rafts, membrane microdomains, membrane regions, transcription factor complexes, neuronal cell bodies, and the apical side of cells.
In the molecular function category, the genes are involved in steroid hormone receptor activity, monocarboxylic acid binding, nuclear receptor activity, transcription factor activity, direct ligand-regulated sequence-specific DNA binding, fatty acid binding, carboxylic acid binding, organic acid binding, and nuclear receptor transcription coactivator activity. KEGG pathway enrichment analysis revealed 118 related pathways. A bubble chart was generated according to the $P$ values and ratios of related genes (Supplemental Table 2, Figure $1(\mathrm{~d})$ ). The bubble chart shows the top 20 pathways, which mainly involved insulin resistance, the adipocytokine signalling pathway, and the PPAR signalling pathway.

3.2. Network Database System Analysis of the Potential Targets of Renshen and Chaihu Herb Pairs. The STRING database was used to establish a PPI network of the intersecting targets of the Renshen and Chaihu herb pair and NAFLD, which involved 63 protein nodes and 351 connections. Topological analysis showed that the median of the twofold degree value of the network was 18 , and 8 targets, AKT1, PPARG, MAPK3, TNF, TP53, SIRT1, STAT3, and PPARA, had degree values greater than or equal to 18 (Figure 2(a)). The median of the twofold degree value of this PPI network was 18 , which conformed to the requirements of the 8 targets: AKT1, PPARG, MAPK3, TNF, TP53, SIRT1, STAT3, and PPARA. On the basis of the herb pair active componentpotential target network, we then determined whether the active components acted on these targets and constructed a regulatory network of components and core targets using Cytoscape 3.7.2 software to visualize any relationship (Figure 2(b)). The network contained 30 nodes and 48 connections, and 28 pairs of connections were found for the components of the Renshen and Chaihu herb pair and the targets.

The 3D structures of AKT1, PPARG, MAPK3, TNF, TP53, SIRT1, STAT3, and PPARA were obtained from the PDB database, and the 3D structure of each monomer component in 


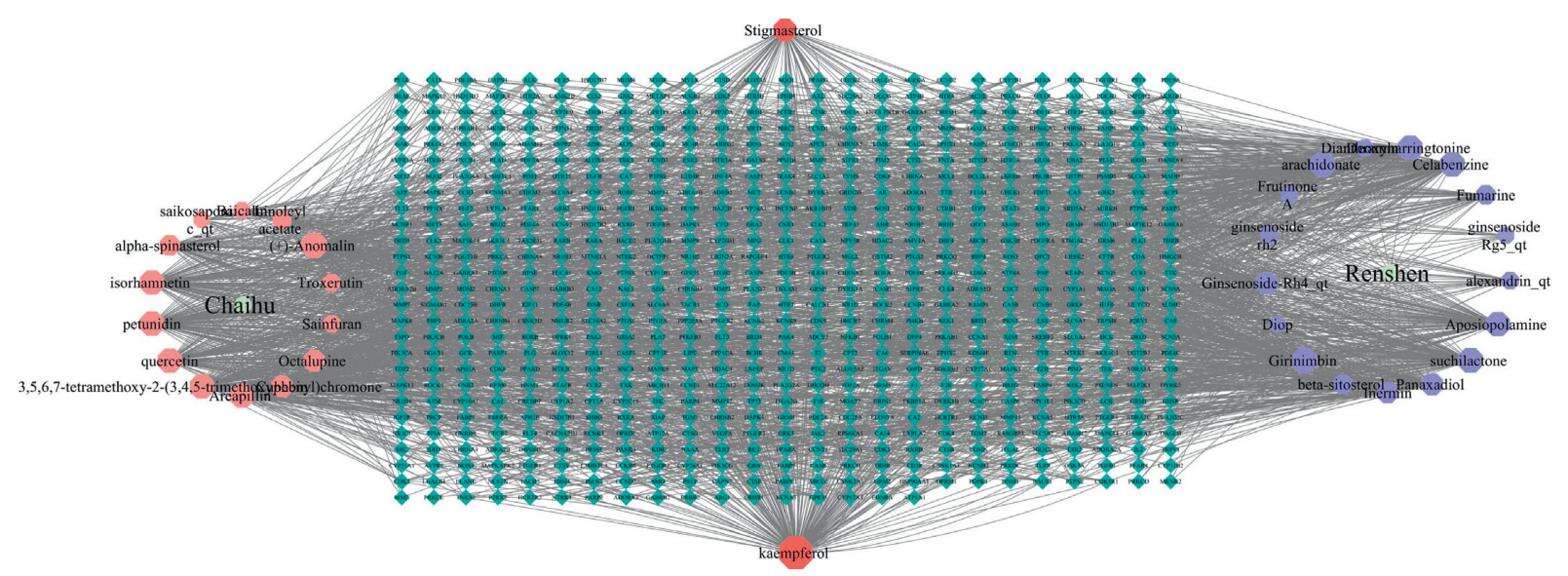

(a)



(b)

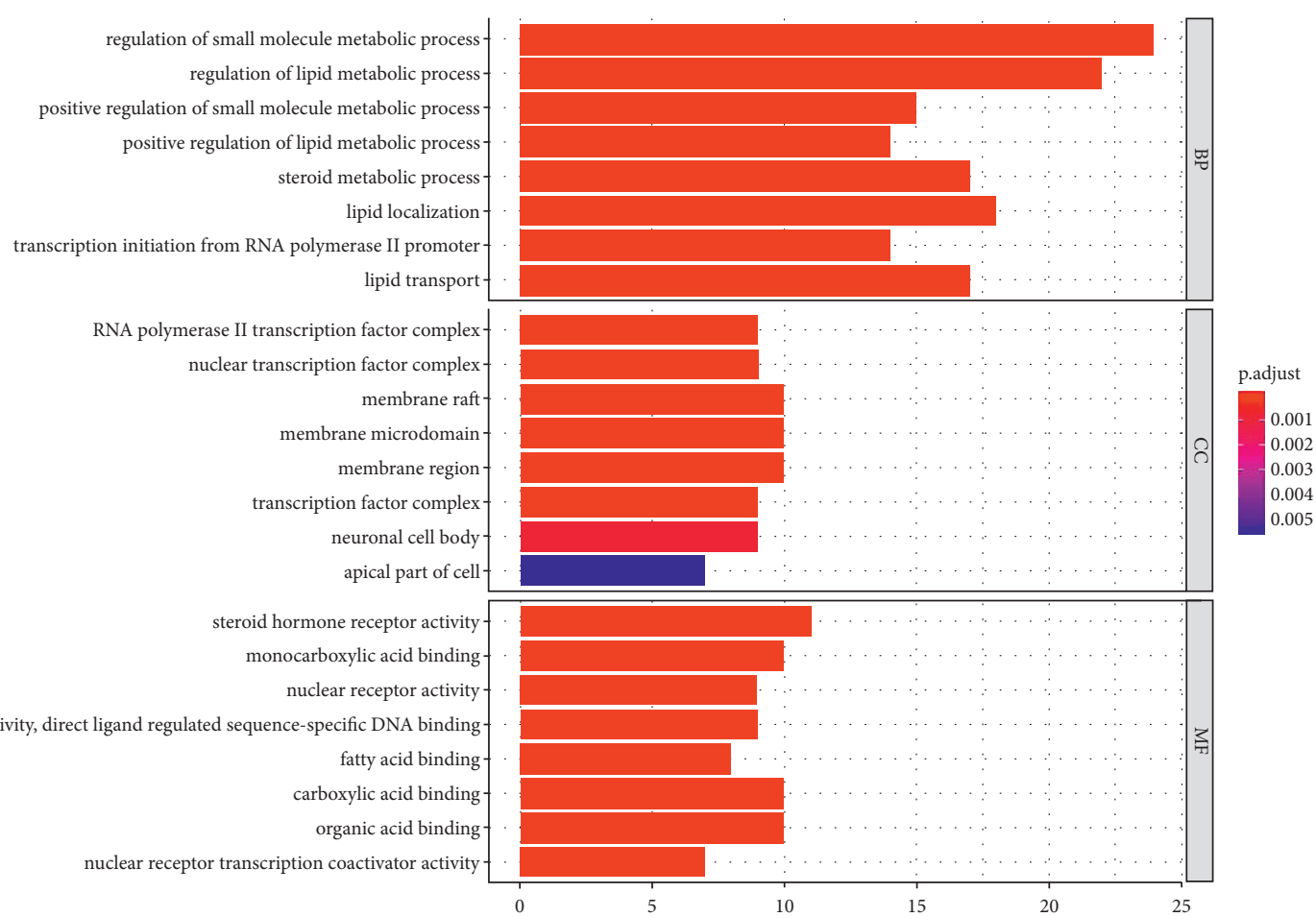

(c)

Figure 1: Continued. 


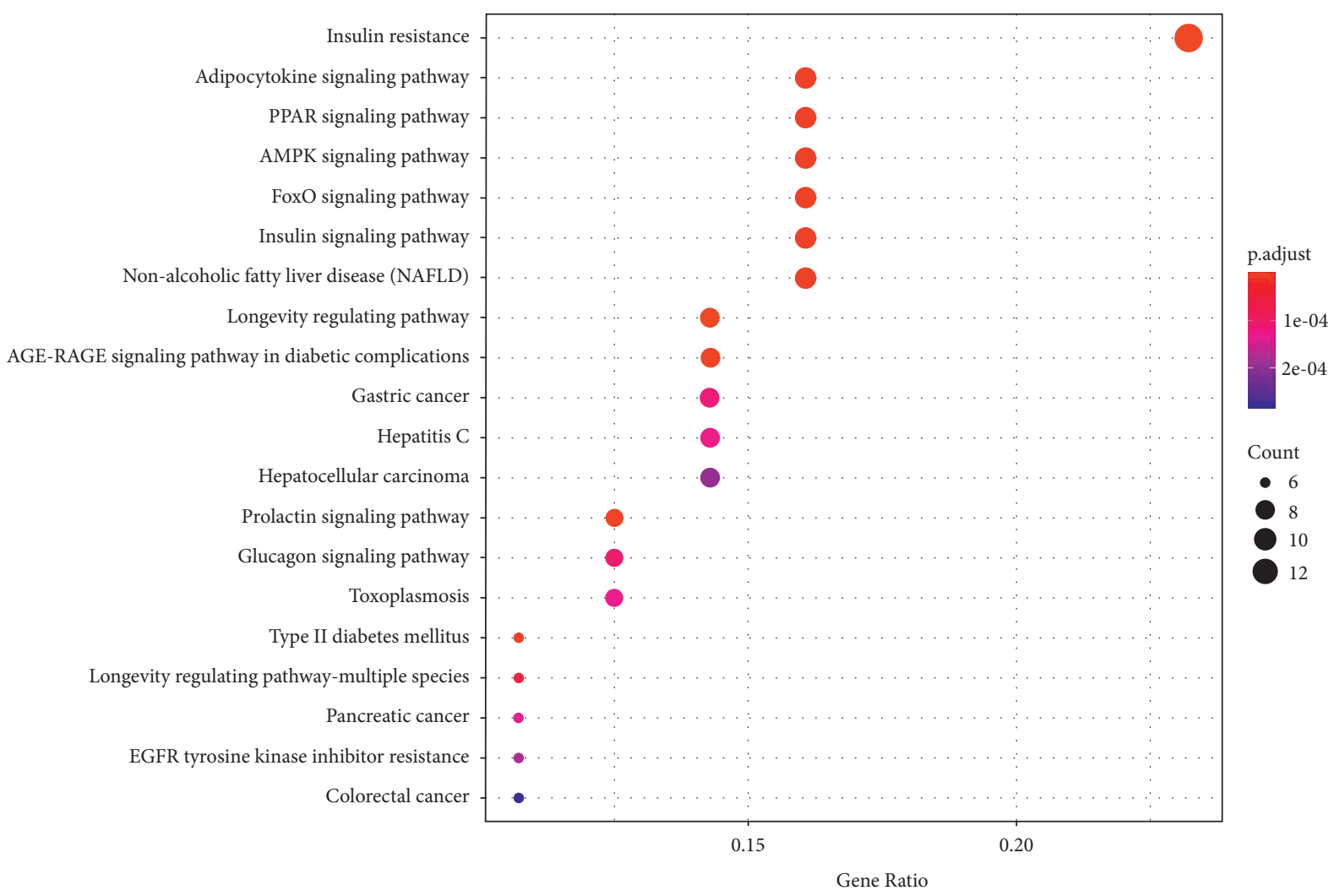

(d)

Figure 1: Network database analysis of the effective material basis of the Renshen and Chaihu herb pair. (a) Target biological network of the Renshen and Chaihu herb pair. (b) Venn analysis of the Renshen and Chaihu herb pair and NAFLD targets. (c) GO enrichment analysis of intersecting targets. (d) KEGG pathway analysis of intersecting targets.

mol2 format was obtained from the ZINC and TCMSP databases. We processed ligands and receptors with hydrogenation and calculated the charge and other steps for use in AutoDock Vina software of semiflexible docking to identify the interaction between the ligands and protein amino acid residues of the proteins. Lower binding energies indicated better docking (Table 3). According to the results of the molecular docking analysis and related literature, we focused on the relationship between stigmasterol and PPAR $\gamma$ (Figure 2(c)).

\subsection{Stigmasterol Reduces Lipid Accumulation in Cells Treated} with Free Fatty Acids. To rule out the cytotoxicity of stigmasterol to HepG2 cells, $0-1000 \mu \mathrm{M}$ stigmasterol was used to treat HepG2 cells for 24 hours and 48 hours, and the results showed no obvious cytotoxicity (Figure 3(a)). HepG2 cells were treated with different concentrations of stigmasterol for 24 hours, stained with Oil Red $\mathrm{O}$ and imaged using a microscope. Compared with group $\mathrm{C}$, more coloured lipid droplets were deposited in the cells of group $\mathrm{M}$, and the rate of lipid change was significantly higher in group $\mathrm{M}$ than in group $\mathrm{C}$. The number of lipid droplets was decreased after stigmasterol treatment (Figure 3(b)). Compared with group C, the TG content increased in group $\mathrm{M}(P<0.01)$. After low-dose stigmasterol intervention, the TG content did not significantly change $(P>0.05)$. After high-dose stigmasterol intervention, the TG content significantly decreased, and the difference was statistically significant $(P<0.01)$ (Figure $3(\mathrm{c}))$. Compared to group $\mathrm{C}$, Nile red staining of HepG2 cells showed that the lipid content in group $M$ was significantly increased and that stigmasterol treatment was significantly more effective in group M (Figure 3(d)).

3.4. Stigmasterol Activates the Expression Level of PPAR $\gamma$ and Downstream UCP-1. RT-PCR showed that compared to those in group C, the mRNA levels of PPAR $\gamma, \mathrm{CPT}-2$, and UCP-1 in group $M$ were significantly reduced $(P<0.05)$. After treatment with low-dose stigmasterol and high-dose stigmasterol, the mRNA levels of PPAR $\gamma, \mathrm{CPT}-2$, and UCP1 were significantly increased $(P<0.05)$ (Figure $4(\mathrm{a}))$. The $\mathrm{WB}$ analysis showed that compared to those in group $\mathrm{C}$, the protein levels of PPAR $\gamma, \mathrm{CPT}-2$, and UCP-1 in group $\mathrm{M}$ were significantly reduced $(P<0.05)$. After treatment with low-dose stigmasterol and high-dose stigmasterol, the protein levels of PPAR $\gamma$ and UCP-1 were significantly increased $(P<0.05)$, but the protein level of CPT-2 did not significantly change $(P>0.05)$ (Figure $4(\mathrm{~b}))$. The immunofluorescence analysis showed that the fluorescence intensity of PPAR $\gamma$ in group $M$ was significantly reduced compared to that in group $\mathrm{C}(P<0.05)$. After stigmasterol treatment, the fluorescence intensity of the PPAR $\gamma$ protein was increased significantly $(P<0.05)$ (Figure $4(\mathrm{c}))$. 


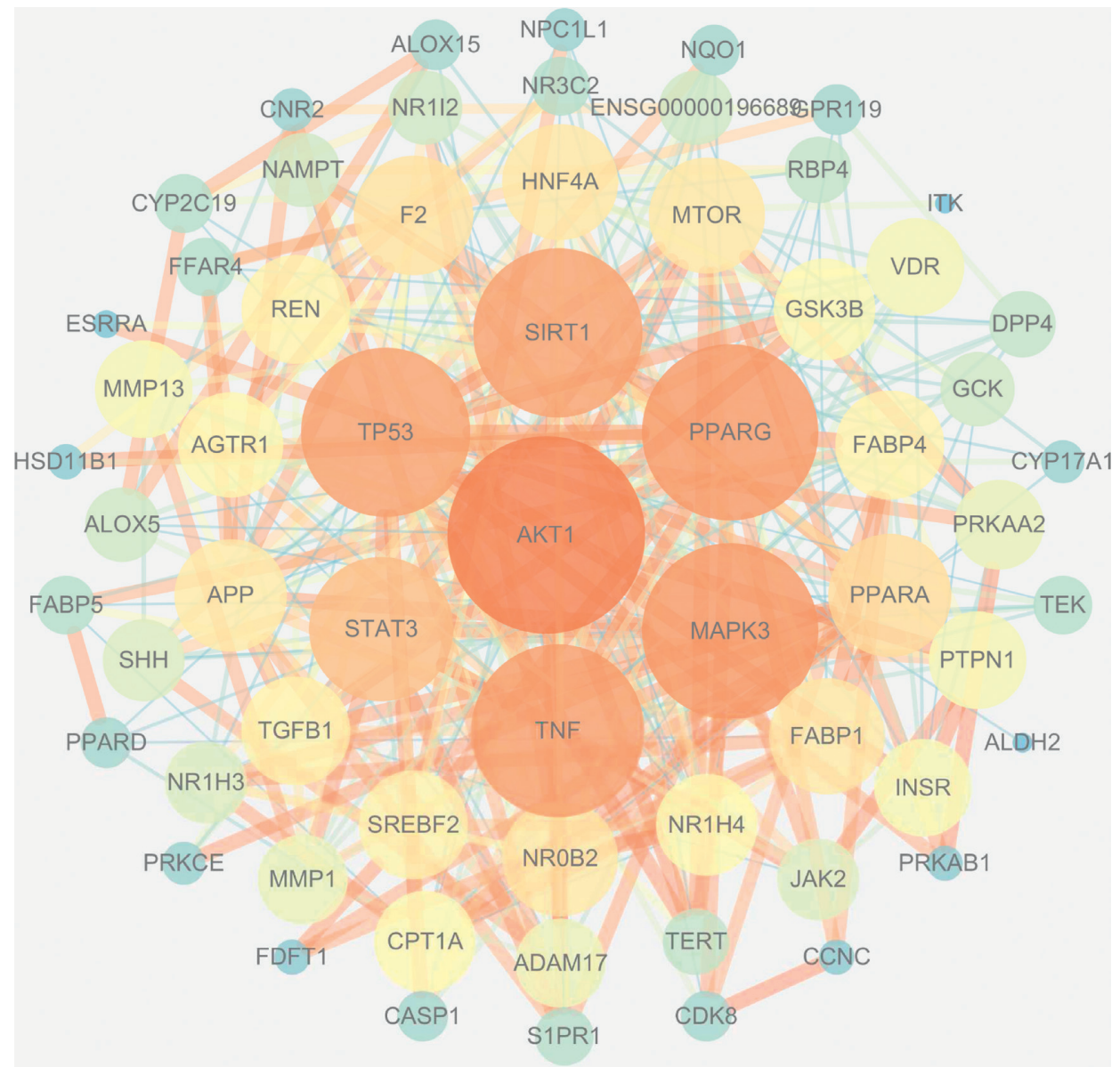

(a)



(b)

FIGURE 2: Continued. 


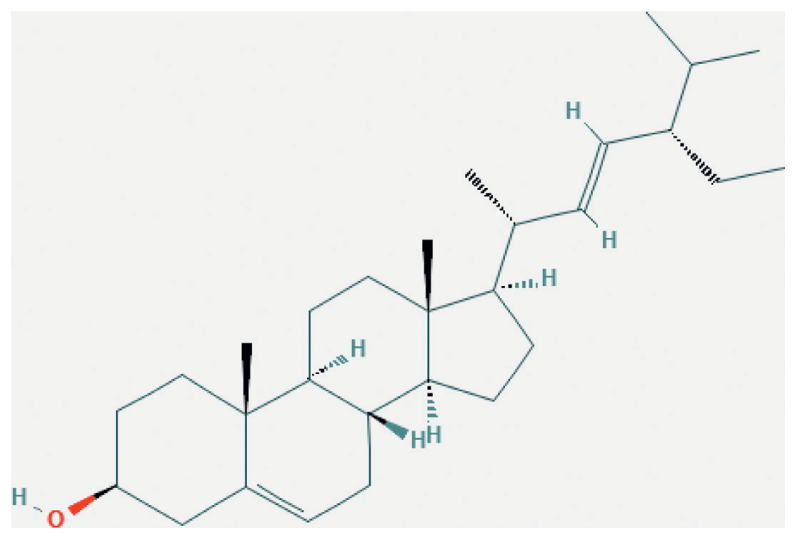

(c)

Figure 2: Network database system analysis of the potential targets of the Renshen and Chaihu herb pair. (a) Construction and analysis of the PPI network of intersecting targets. (b) Renshen and Chaihu herb pair component and core target regulatory network. (c) Molecular structure of stigmasterol.

TABLE 3: Results of docking Renshen and Chaihu herb pair components with core targets.

\begin{tabular}{|c|c|c|}
\hline Target & Renshen and Chaihu herb pair components & Highest binding energy $(\mathrm{kcal} / \mathrm{mol})$ \\
\hline AKT1 & Girinimbin & -10.2 \\
\hline AKT1 & Quercetin & -8.8 \\
\hline AKT1 & Kaempferol & -8.6 \\
\hline AKT1 & Petunidin & -8.6 \\
\hline AKT1 & Areapillin & -8.5 \\
\hline AKT1 & Isorhamnetin & -8.5 \\
\hline AKT1 & 3,5,6,7-Tetramethoxy-2-(3,4,5-trimethoxyphenyl) chromone & -8.4 \\
\hline MAPK3 & $\alpha$-Spinasterol & -9.2 \\
\hline PPARA & Stigmasterol & -8.3 \\
\hline PPARA & Aposiopolamine & -7.2 \\
\hline PPARA & Arachidonate & -7.2 \\
\hline PPARA & $\alpha$-Spinasterol & -7 \\
\hline PPARG & $\alpha$-Spinasterol & -8.1 \\
\hline PPARG & Stigmasterol & -7.8 \\
\hline PPARG & $\beta$-Sitosterol & -7.6 \\
\hline PPARG & Arachidonate & -6.6 \\
\hline PPARG & Ginsenoside $\mathrm{Rh} 4 \mathrm{qt}$ & -6.4 \\
\hline SIRT1 & Fumarine & -8.4 \\
\hline SIRT1 & Sainfuran & -7.6 \\
\hline STAT3 & Ginsenoside rh2 & -6.9 \\
\hline STAT3 & Saikosaponin cqt & -6.8 \\
\hline STAT3 & Ginsenoside $\operatorname{Rg} 5 \mathrm{qt}$ & -6.6 \\
\hline STAT3 & Ginsenoside $\mathrm{Rh} 4 \mathrm{qt}$ & -6 \\
\hline STAT3 & Alexandrine qt & -5.5 \\
\hline TNF & Baicalin & -7.6 \\
\hline TP53 & Arachidonate & $\mathbf{0}$ \\
\hline
\end{tabular}

3.5. Generation of the PPAR $\gamma$-Knockdown Cell Line. The results of docking stigmasterol with $\operatorname{PPAR} \alpha$ and $\operatorname{PPAR} \gamma$ molecules were visualized with PyMOL software, and threedimensional schematic diagrams of the binding of stigmasterol with PPAR $\alpha$ and PPAR $\gamma$ were obtained. The best binding energies were $-8.3 \mathrm{kcal} / \mathrm{mol}$ and $-7.8 \mathrm{kcal} / \mathrm{mol}$, showing that stigmasterol has good binding activity to both PPAR $\alpha$ and PPAR $\gamma$ (Figure 5(a)).

Three cas9-gRNAs and one control empty vector were transfected into HepG2 cells, and puromycin was used for screening 48 hours after transfection. After all the untransfected normal cells died, the adherent cells of the transfection group were collected for WB experiments. The experimental results showed that the greatest knockdown of PPAR $\gamma$ resulted from sgRNA3 transfection (Figure 5(b)). Thus, sgRNA3 was subsequently used to generate $\operatorname{PAAR} \gamma$-knockdown cells for the drug intervention experiments.

3.6. Knockdown of PPARY Reverses the Effect of Stigmasterol on Blood Lipid Reduction. After stigmasterol treatment, Oil Red $\mathrm{O}$ staining showed that compared to the $\operatorname{PPAR} \gamma^{-/}$group, 


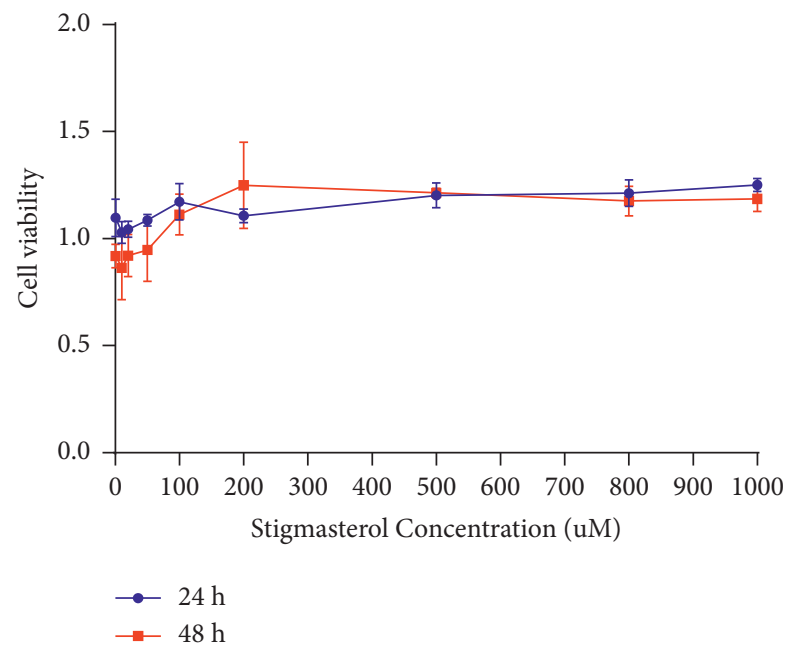

(a)

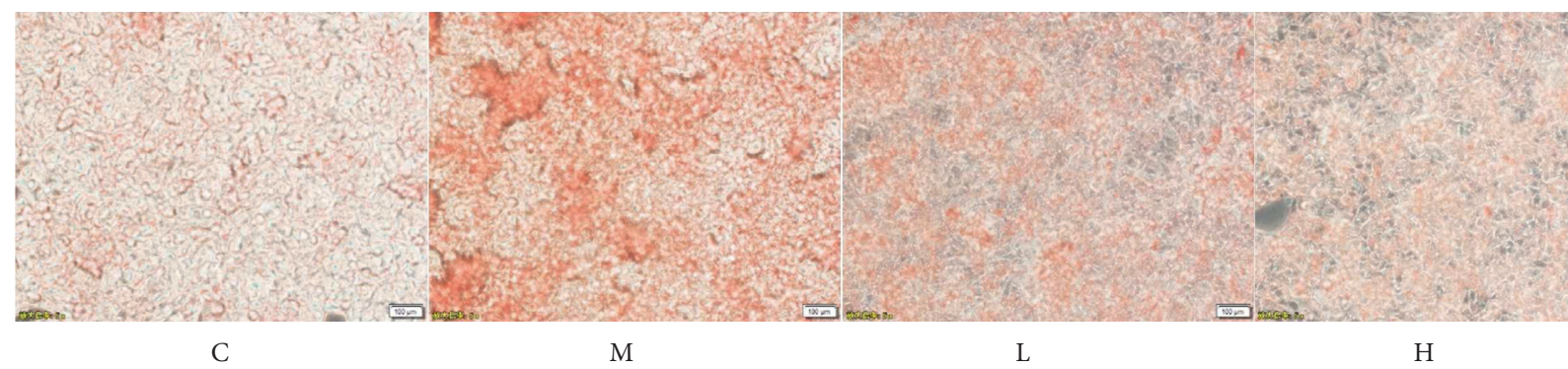

(b)

HepG2

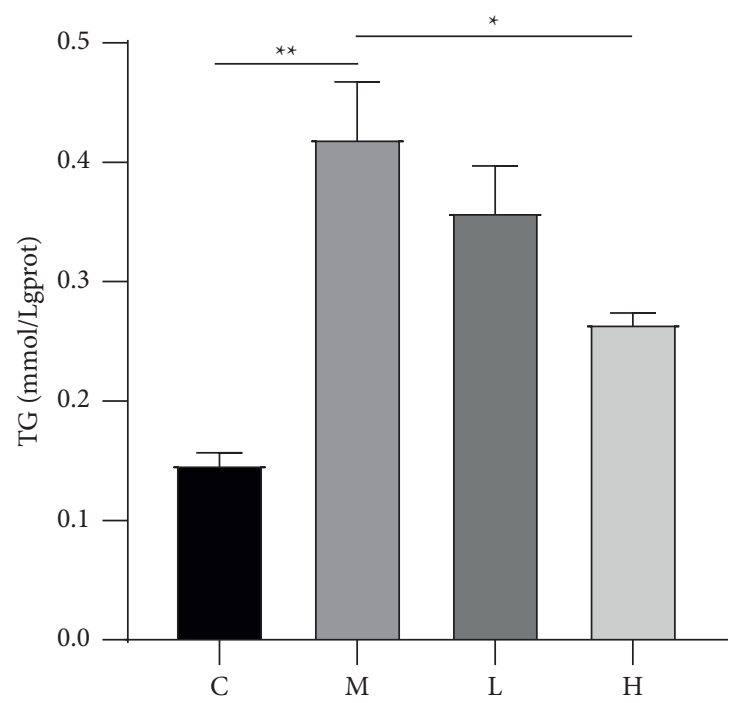

(c)

Figure 3: Continued. 


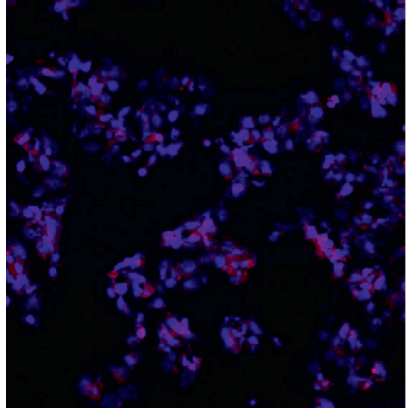

C

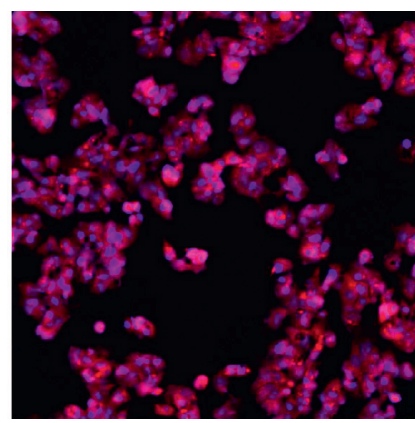

$\mathrm{M}$

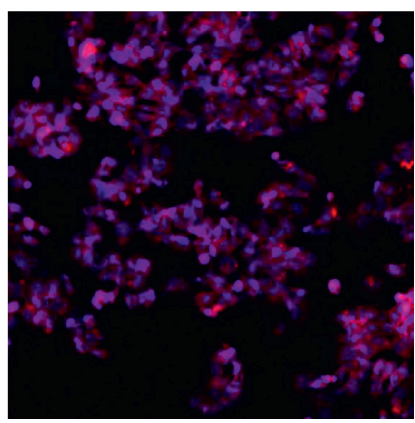

$\mathrm{H}$

(d)

FiguRE 3: Stigmasterol reduces lipid accumulation in cells treated with free fatty acids. Cells were treated as described in the "Methods" section. After treatment, cells were harvested. (a) Cytotoxicity was detected by a WST-1 kit. (b) A total of $400 \mu \mathrm{M}$ oleic acid, $200 \mu \mathrm{M}$ palmitic acid, or $30 \mu \mathrm{M} / 60 \mu \mathrm{M}$ stigmasterol was used to treat AML12 cells and HepG2 cells, respectively. Oil Red O test to detect the degree of cell fat change. (c) The TG level was detected by a kit and then normalized, as shown in the bar graph. (d) Nile red staining was imaged using a laser confocal microscope. ${ }^{*} P<0.05$ and ${ }^{* *} P<0.01$ compared to group $C^{*} P<0.05$ and ${ }^{* *} P<0.01$ compared to group M.
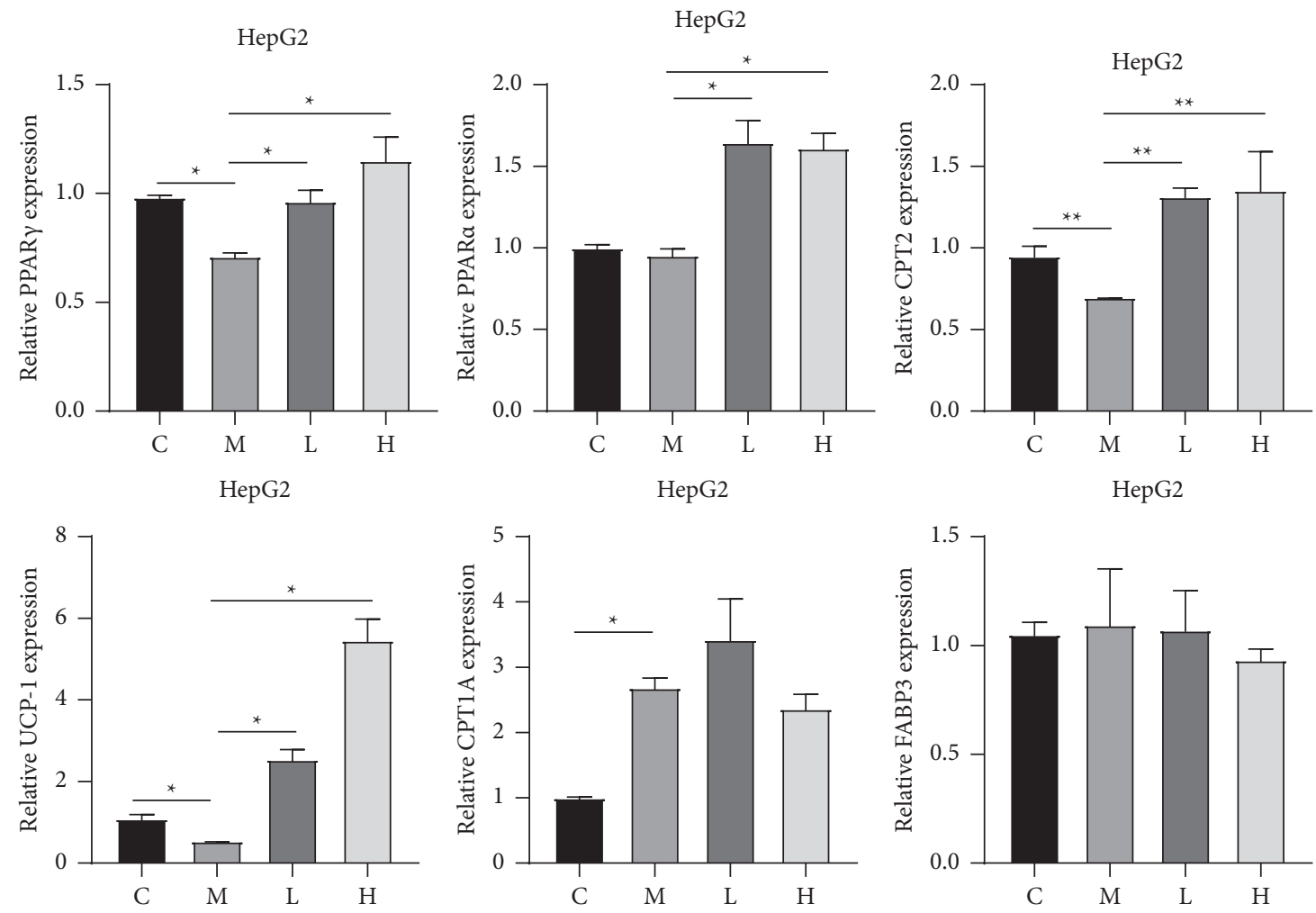

(a)

FIgUre 4: Continued. 

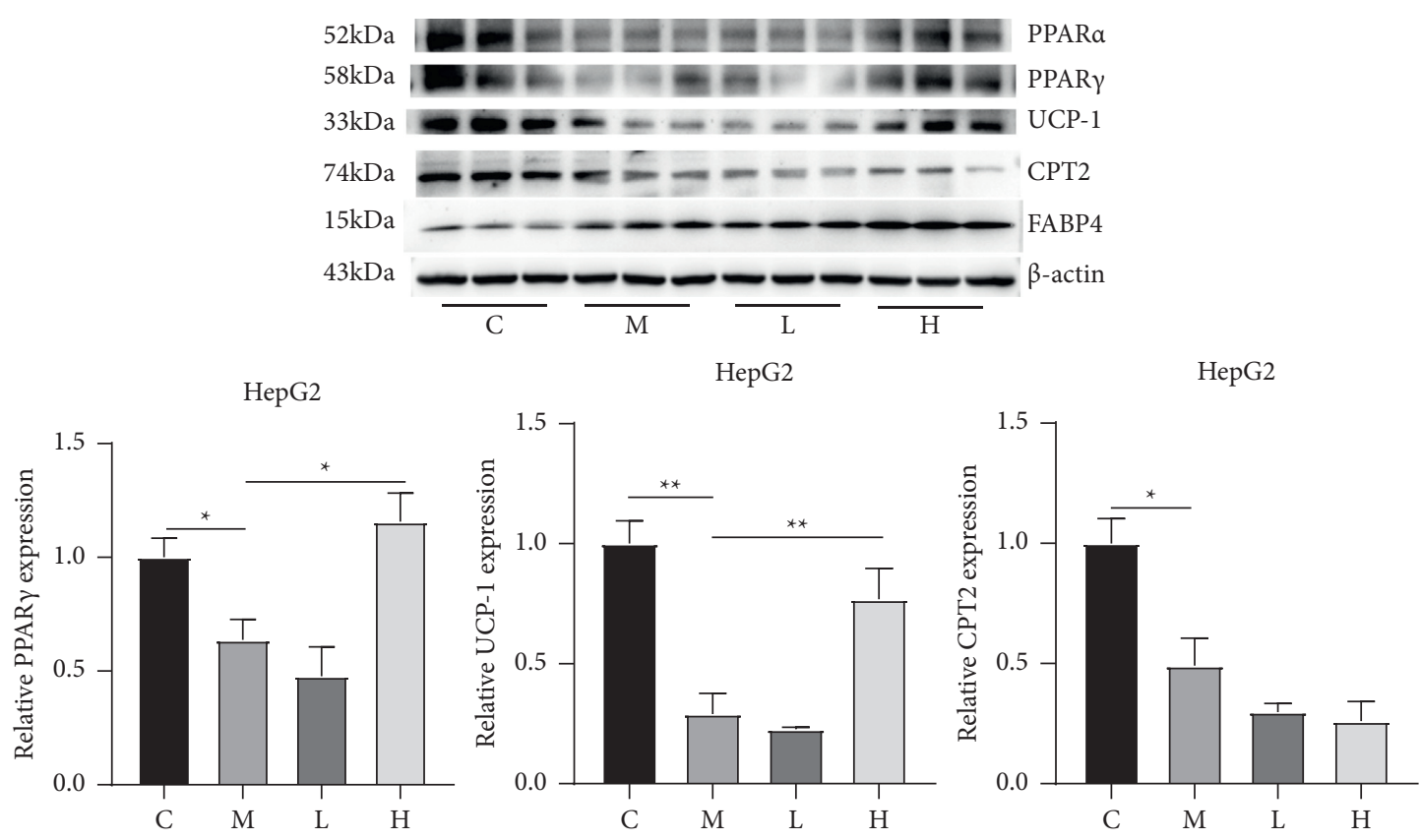

HepG2

HepG2
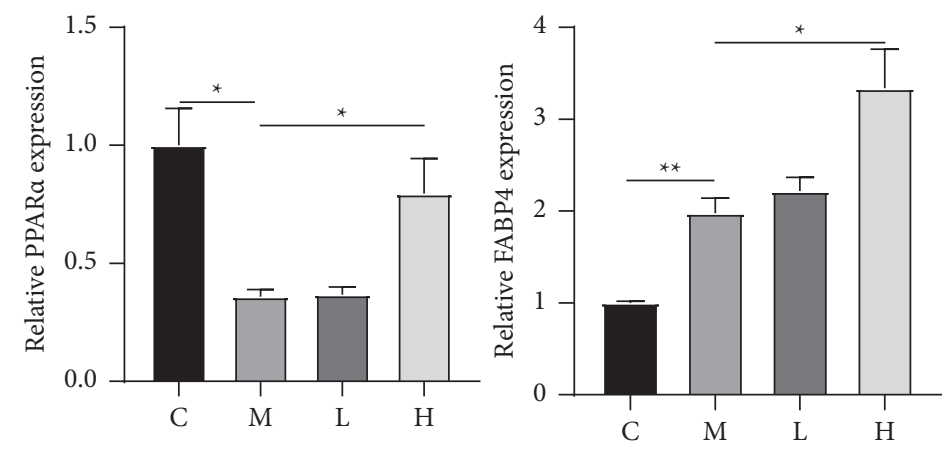

(b)

DAPI
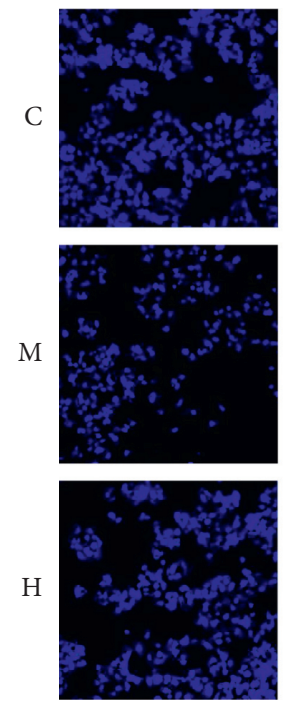

PPAR $\gamma$
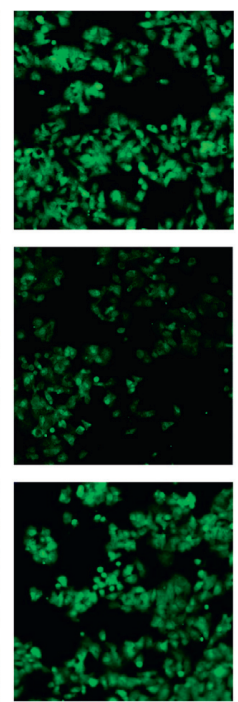

Merge
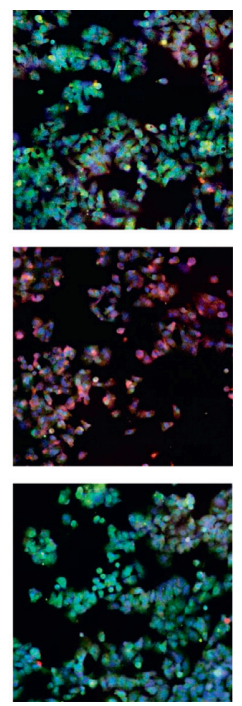

DAPI
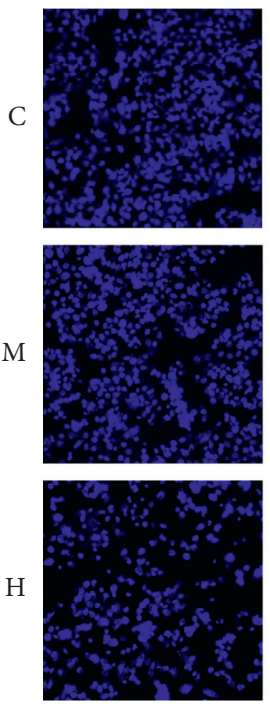

PPARa
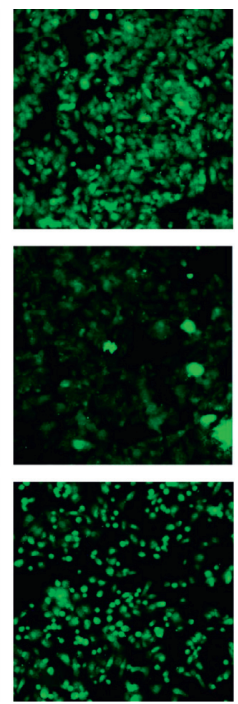

Merge
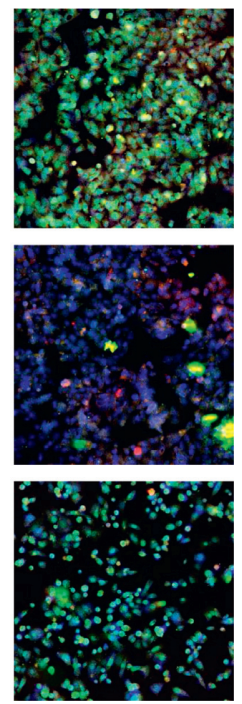

(c)

Figure 4: Stigmasterol activates PPAR $\gamma$ and its downstream targets. Cells were treated as described in the "Methods" section. After treatment, the cells were harvested. (a) RT-PCR was performed to detect the mRNA expression levels of PPAR $\gamma$, CPT-2, and UCP-1. (b) Western blot analysis was performed to detect the protein expression levels of PPAR $\gamma$, CPT-2, and UCP-1. (c) Immunofluorescence was used to detect the protein level of PPAR $\gamma$ and PPAR $\alpha .{ }^{*} P<0.05$ and ${ }^{* *} P<0.01$ compared to group $\mathrm{C}^{*} P<0.05$ and ${ }^{* *} P<0.01$ compared to group $\mathrm{M}$. 


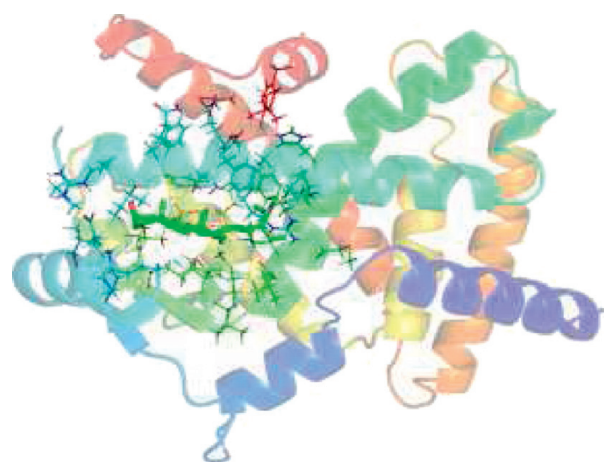

(a)
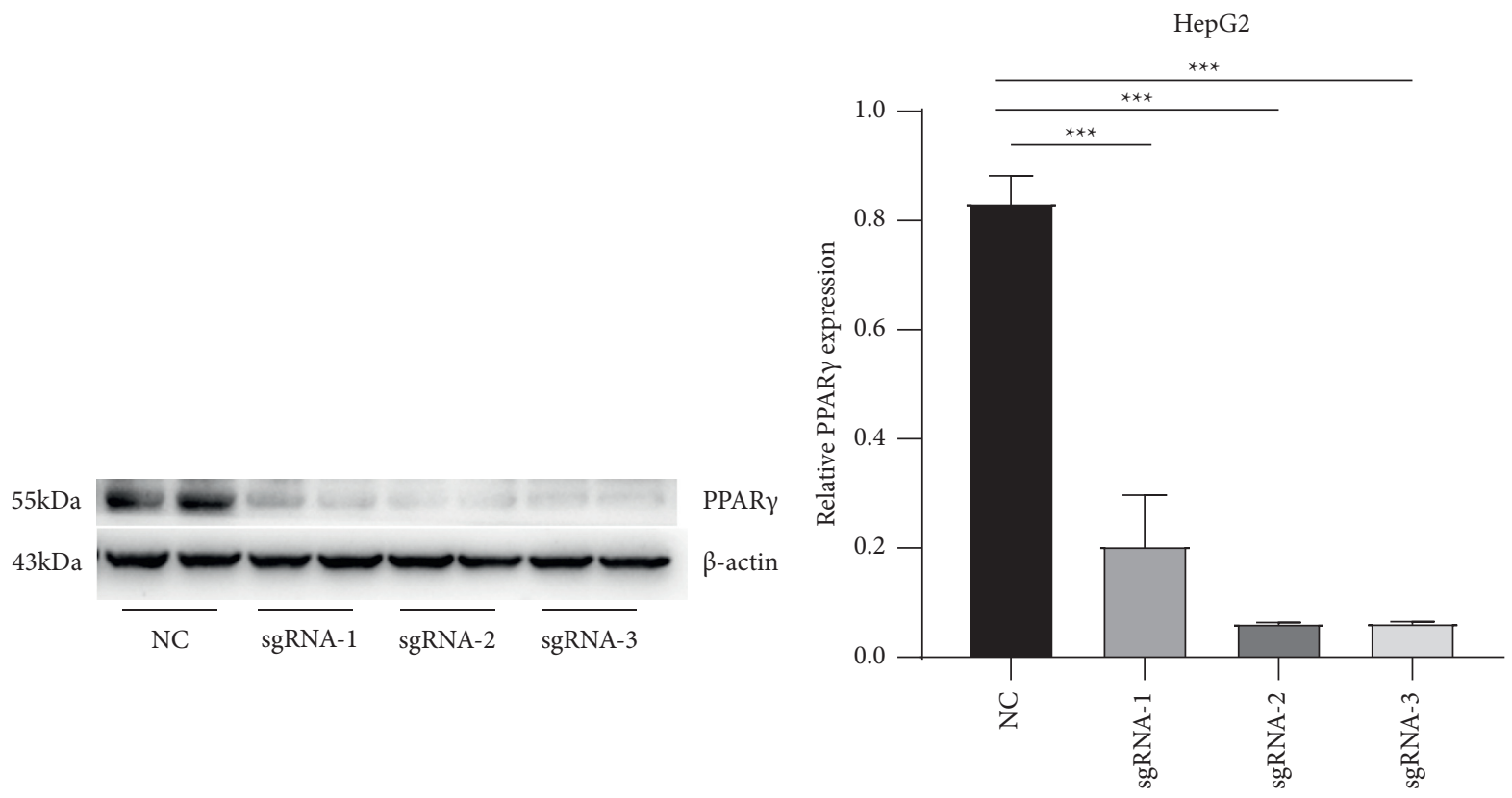

(b)

Figure 5: Generation of the PPAR $\gamma$-knockdown cell line. Knockdown cells were generated as described in the "Methods" section. (a) Schematic diagram of the docking activity of PPAR $\gamma$ and stigmasterol. The binding free energy activity was $-7.8 \mathrm{kcal} / \mathrm{mol}$. (b) The PPAR $\gamma$ gene in HepG2 cells was knocked down by transfecting a PPAR $\gamma$ CRISPR/Cas9 plasmid. The transfected cells were screened with puromycin, and total cell protein was extracted for Western blot analysis to detect PPAR $\gamma$ protein expression. ${ }^{*} P<0.05$, ${ }^{* *} P<0.01$, and ${ }^{* * *} P<0.001$ compared to the PPAR $\gamma^{-/-}$group. ${ }^{*} P<0.05,{ }^{* *} P<0.01$, and ${ }^{* * *} P<0.001$ compared to the PPAR $\gamma^{-/-}$group.

the PPAR $\gamma^{-/-}+$STI group showed no significant changes in the deposition of coloured lipid droplets, and there was no significant difference in the rate of lipid level change (Figure 6(a)). Nile red staining showed that compared to the PPAR $\gamma^{-/}$group, the lipid content in the PPAR $\gamma^{-/-}+$STI group did not significantly change (Figure 6(b)). Compared to the PPAR $\gamma^{-/}$group, the TG content in the PPAR $\gamma^{-/-}+$STI group did not significantly change $(P>0.05)$ (Figure $6(\mathrm{c}))$. The PCR analysis showed that compared to the PPAR $\gamma^{-/-}$group, the mRNA levels of PPAR $\gamma$ and UCP-1 in the PPAR $\gamma^{-/-}+$STI group did not significantly change $(P>0.05)$ (Figure $6(\mathrm{~d}))$. The WB analysis showed that compared to the PPAR $\gamma^{-/-}$group, the PPAR $\gamma^{-/-}$ + STI histone levels of PPAR $\gamma$ and UCP-1 did not significantly change $(P>0.05)$ (Figure 6(e)). The immunofluorescence analysis showed that compared to the PPAR $\gamma^{-/-}$group, the $\operatorname{PPAR} \gamma$ protein fluorescence intensity in the $\operatorname{PPAR} \gamma^{-/-}+\mathrm{STI}$ group did not significantly change $(P>0.05)$ (Figure 6(f)).

\section{Discussion}

In recent years, researchers have studied the pharmacological properties of the Renshen and Chaihu herb pair in the treatment of metabolic diseases, especially NAFLD. Radix Bupleuri mainly plays a role in anti-inflammation, liver protection, and immune regulation [19-21]. Radix Ginseng is beneficial in improving glucose metabolism, lipid metabolism, liver function, and oxidative stress, and it has antifibrotic effects [22-24].

To investigate the pharmacodynamic mechanism of the Renshen and Chaihu herb pair in the treatment of metabolic diseases, our research group analysed the drug pair of the Renshen and Chaihu herb pair through network pharmacology and molecular docking technology. The results showed that stigmasterol is a common active ingredient in the Renshen and Chaihu herb pair, and the results suggested 


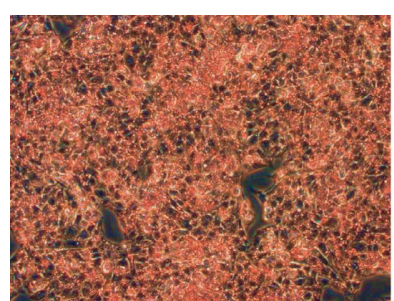

PPAR $\gamma^{-/-}$

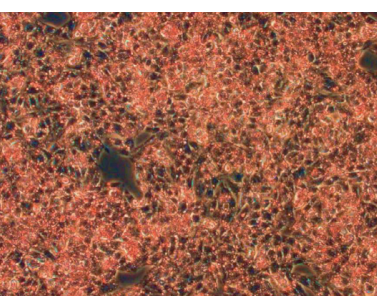

PPAR $\gamma^{-/-}+$STI

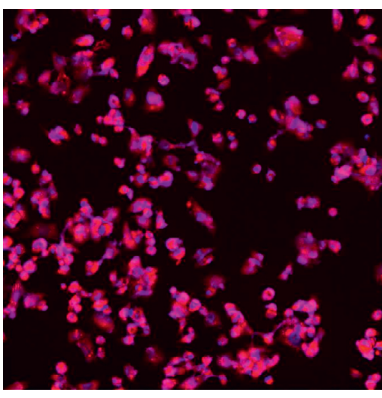

PPAR $\gamma^{-/-}$

(a)

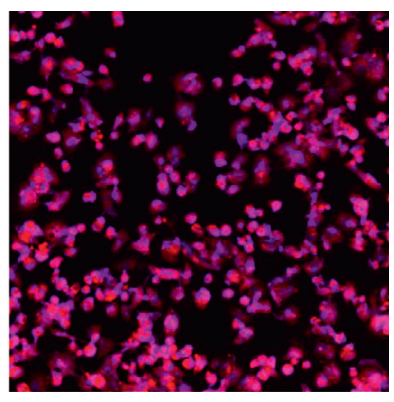

PPAR $\gamma^{-/-}+$STI

(b)

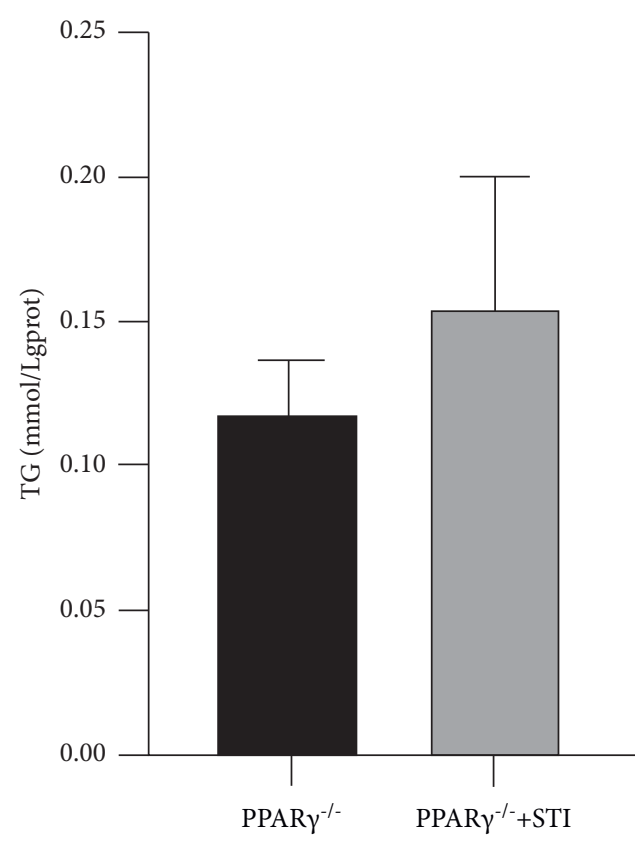

(c)

HepG2

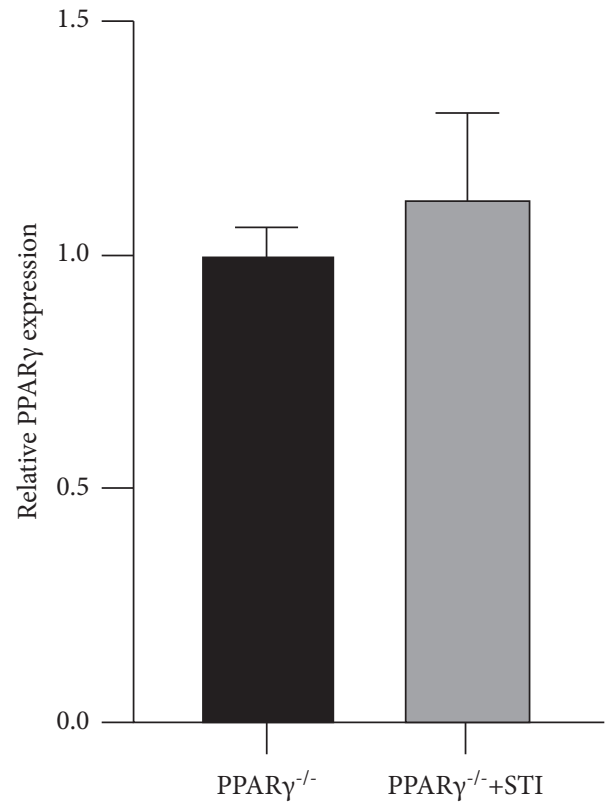

HepG2

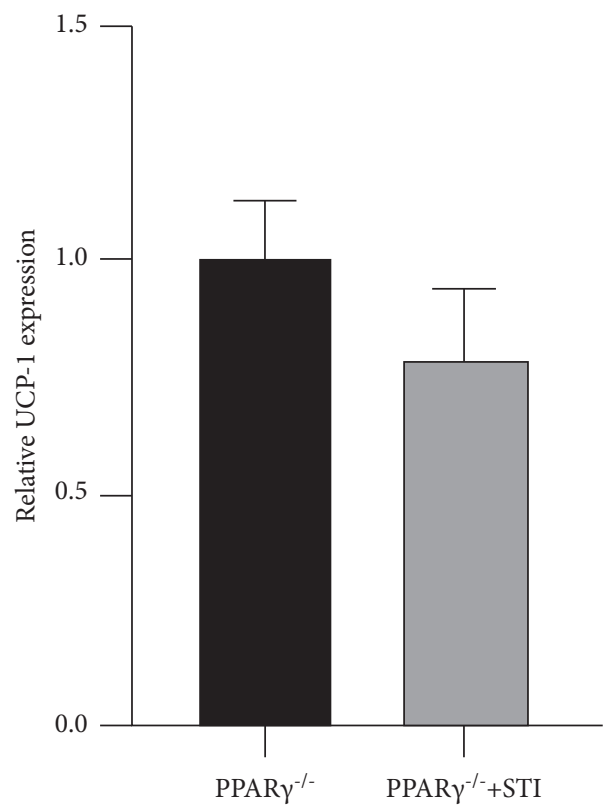

(d)

Figure 6: Continued. 

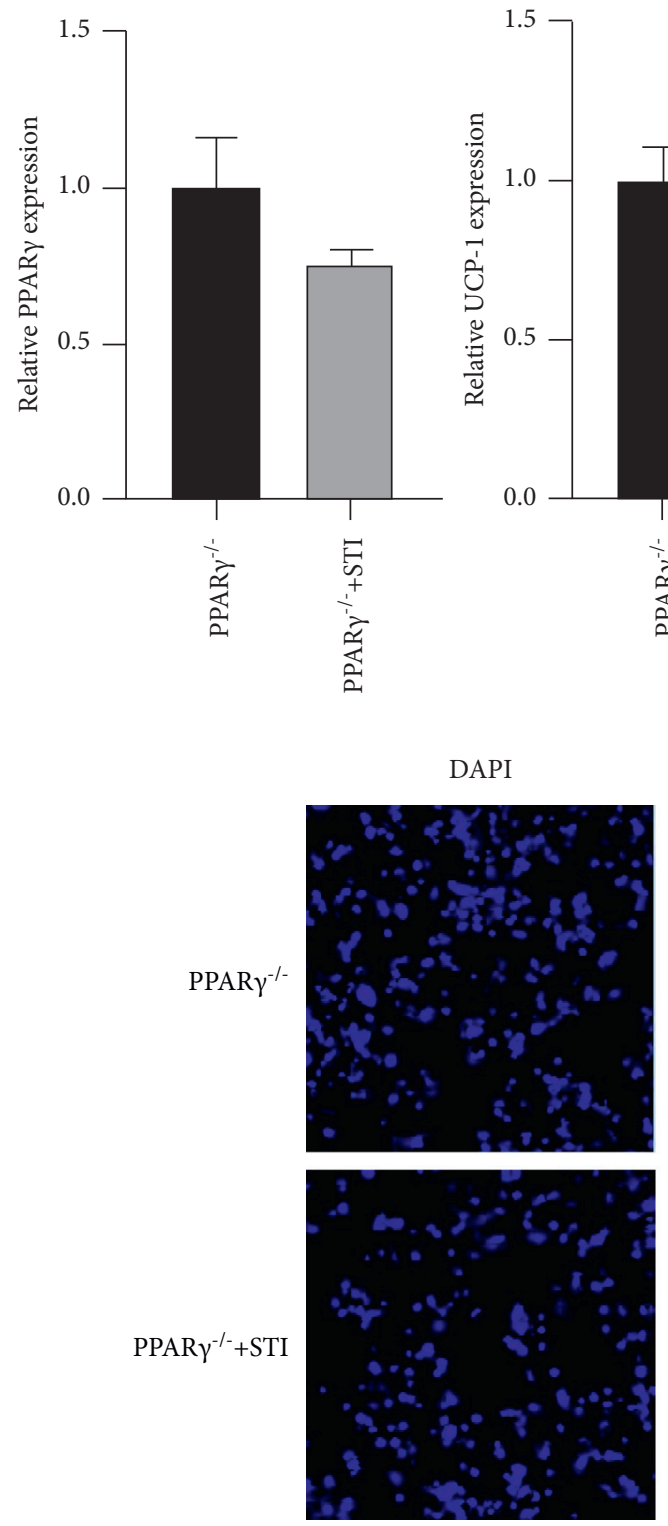

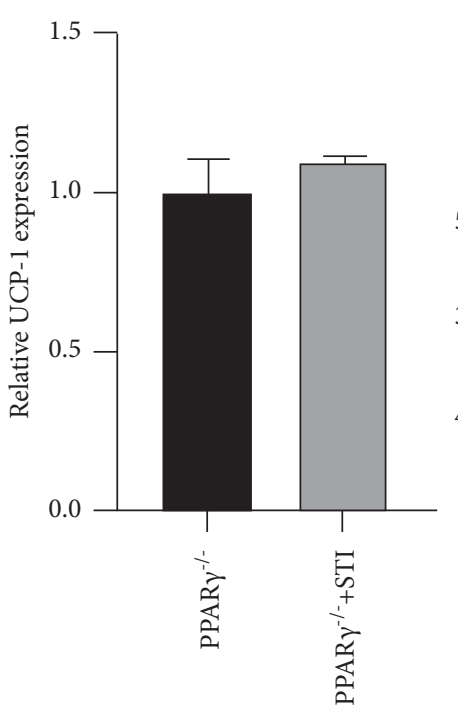

(e)
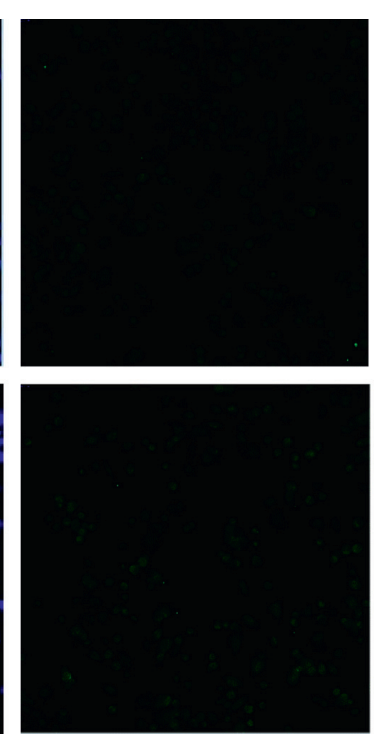

(f)

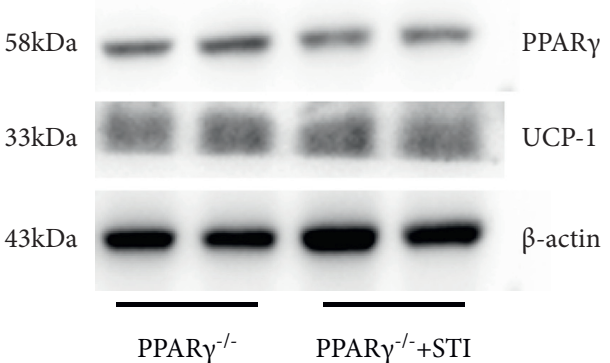

PPAR $\gamma^{-1-2} \quad$ PPAR $\gamma^{-1}+\mathrm{STI}$
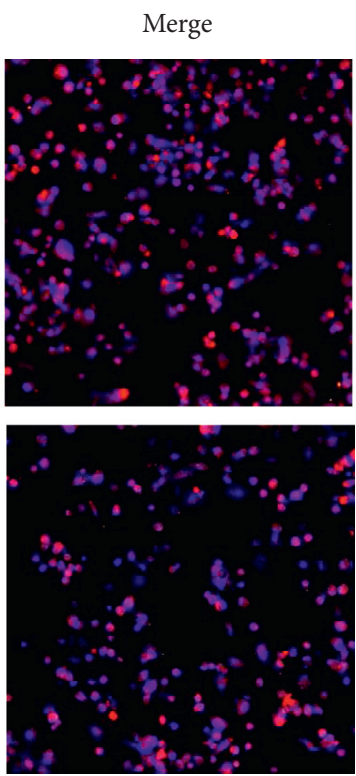

FIGURE 6: After knocking down PPAR $\gamma$, the lipid-lowering effect of stigmasterol was reversed. Treat the cells as described above. After the treatment, the cells were harvested. (a) Oil Red O experiment to detect the lipid accumulation level of cells in the PPAR $\gamma^{-/-}$group and PPAR $\gamma^{-1-}+$ STI group. (b) Nile red stained, observed under a laser confocal microscope to obtain an image. (c) Detect the TG level by the kit and normalize it to the TG level. (d) RT-PCR to detect the mRNA expression level of PPAR $\gamma$ and UCP-1. (e) Western blot detection of the protein expression level of PPAR $\gamma$, UCP-1. (f) Using immunofluorescence technology to detect the protein level of PPAR $\gamma$. Compared with the PPAR $\gamma^{-/-}$group, ${ }^{*} P<0.05$ and ${ }^{* *} P<0.01$.

that PPAR $\gamma$ may be a potential target. Importantly, antagonizing PPAR $\gamma$ has previously been shown to attenuate NAFLD. Therefore, for this study, we hypothesized that stigmasterol, the active ingredient of the Renshen and Chaihu herb pair, may exert its therapeutic effect by activating PPAR.

The pathogenesis of NAFLD is complicated, and existing theories do not fully explain it. In the past, the "second hit" theory was the most popular explanation of NAFLD pathogenesis. Recently, an increasing number of possible mechanisms have been discovered that promote the development of NAFLD, including the production of lipotoxic lipids, the activation of inflammasomes, insulin resistance, intestinal flora imbalance, and hepatocyte fibrosis [25-29].

Therefore, to regulate glucose metabolism and lipid metabolism homeostasis, as well as to attenuate liver cell oxidative stress, improve mitochondrial energy metabolism, and reduce inflammation and fibrosis, researchers have sought a series of targets for the treatment of NAFLD. However, there are no specific drugs for NAFLD treatment. Insulin sensitizers, SGLT-2 inhibitors, lipid-lowering drugs, 
and antioxidants have shown certain effects in mitigating NAFLD, but there are also certain limitations to the use of these treatments, as revealed in previous studies: the insulin sensitizer, rosiglitazone, attenuates liver steatosis, hepatocyte inflammation, and fibrosis in patients with NASH [30]. Metformin improves measures of fasting blood glucose, insulin resistance, and serum adiponectin in NAFLD patients [31], but these results are controversial [32]. The SGLT-2 inhibitor, empagliflozin, reduces liver fat accumulation and lowers alanine aminotransferase levels in NAFLD patients [33]. Obeticholic acid, a selective FXR agonist, mitigates liver fibrosis in patients with NASH [34]. The antioxidant, vitamin E, attenuates liver steatosis, lobular inflammation, hepatocyte ballooning, and liver fibrosis in patients with NASH [35], but long-term use of vitamin E may increase the risk of stroke and prostate cancer [6]. Berberine reduces the levels of TG, TC, and LDL-c in patients with NAFLD, and it has a certain effect on liver function and blood sugar [36]. Pentoxifylline ameliorates liver steatosis, lobular inflammation, and fibrosis in patients with NASH [37]. Curcumin reduces the levels of alanine aminotransferase and aspartate aminotransferase by regulating the levels of total cholesterol and triglycerides to improve NAFLD [38]. Green cardamom has shown certain advantages in improving blood sugar and fatty liver in obese NAFLD patients [39].

Peroxisome proliferator-activated receptor (PPAR) belongs to the nuclear hormone receptor superfamily, which is divided into the following three subtypes: $\operatorname{PPAR} \alpha, \operatorname{PPAR} \beta / \delta$, and PPAR $\gamma$. PPAR $\alpha$ has been previously shown to play a role in regulating circulating lipids or cellular lipids by regulating liver and skeletal muscle lipid metabolism [40], and it is involved in peroxisome and mitochondrial $\beta$-oxidation, as well as FA transport and glucose production [41]. PPAR $\beta / \delta$ has been shown to participate in lipid oxidation and cell proliferation. A previous study showed that $\operatorname{PAAR} \gamma$ promotes adipocyte differentiation, and it is related to glucose metabolism and lipid uptake; PPAR $\gamma$ also regulates the gene expression of glucose transporter 4 (GLUT4) and adiponectin (ADIPOQ) [42].

Downstream molecules of $\operatorname{PPAR} \gamma$ regulate the transcription of multiple genes. Pyruvate dehydrogenase kinase 4 (PDK4) is a member of the PDK/BCKDK protein kinase family, which inhibits the pyruvate dehydrogenase complex by phosphorylation of its subunits, and it also has been previously shown to participate in apoptosis and insulin resistance [43]. Acyl-CoA oxidase 1 (ACOX1) is involved in the fatty acid $\beta$-oxidation pathway. CD147 has been previously shown to inhibit fatty acid $\beta$-oxidation by activating the p38 MAPK signalling pathway, thereby downregulating $\operatorname{PPAR} \alpha$ and its transcription target genes, namely, carnitine palmitoyltransferase 1A (CPT1A) and ACOX1 [44]. MaR1 also has been previously found to induce AMPK phosphorylation in DIO mice and primary hepatocytes, and AMPK inhibition completely blocks the effect of MaR1 on the expression of CPT1A, ACOX1, ATG5, and ATG7, providing a new treatment for ameliorating fatty liver [45]. Studies have also shown that LPIN2 and ST3GAL5 are new $\mathrm{PPAR} \beta / \delta$ target genes and that the upregulation of $\mathrm{PPAR} \beta / \delta$ gene expression is sensitive to plasma FFA levels [46]. Another study has shown that when CD36 decreases, adiponectin and uncoupling protein 2 (UCP2) increase [47]. Shenqi granules significantly reduce serum TC, TG, and LDL-C levels in a previous study, and they mediate FA uptake, affect mitochondrial energy metabolism, promote the tricarboxylic acid cycle, promote the transport of ATP from mitochondria to the cytoplasm, and restore mitochondrial function [48]. UCP-1 is also downstream of the three subtypes of $\operatorname{PPAR} \alpha, \operatorname{PPAR} \beta / \delta$, and $\operatorname{PPAR} \gamma$, and it is highly expressed in brown adipose tissue. UCP-1 is a transmembrane channel protein located on the inner mitochondrial membrane, and it is the final effector molecule of brown adipose tissue in the process of heat production. For example, a previous study has suggested that after activation, UCP1 in the mitochondria that originally produced ATP is uncoupled and enters the mitochondrial matrix from the mitochondrial membrane space, and a large amount of heat is generated in the process, which is called adaptive thermogenesis [49]. After the sympathetic nervous system is stimulated by stress, protein kinase A was previously shown to activate downstream phosphorylation, promote the cysteine oxidation of UCP1, and activate its uncoupling properties to exert a thermogenic effect [50]. Therefore, activating UCP-1 has been suggested to exert a positive effect on weight loss, preventing hypertension and improving oxidative stress and other metabolic states [51]. When UCP1 expression decreases, mitochondrial ROS increases, which may lead to insulin resistance in patients with type 2 diabetes.

In view of the multiple beneficial effects of PPAR in metabolism, seeking PPAR agonists has always been a hot research topic in NAFLD. However, the clinically used PPAR $\alpha$ agonists, fibrates, have not shown a significant improvement in NAFLD. In addition, a previously performed meta-analysis has shown that the PPAR $\gamma$ agonist pioglitazone has a beneficial effect on advanced fibrosis in patients with NASH [52]. Pioglitazone can improve liver steatosis, lobular inflammation, and balloon dilatation, but its effect on weight gain and its inability to increase the improvement rate of NAFLD limit its further development. Therefore, an increasing number of researchers are devoted to the development of dual or multiple agonists of PPAR. Studies have shown that saroglitazar, a dual agonist of $\operatorname{PPAR} \alpha / \gamma$, improves insulin resistance and steatohepatitis in NASH mice [53]. Studies have also reported that the PPAR pan-agonist can reduce steatosis, reverse liver injury and monocyte infiltration, and reduce the inflammatory response in macrophages and monocytes with the participation of PPAR $\delta$ [54].

Phytosterols are widely distributed in a variety of plant cells, including vegetable oils, plant seeds, and pollen, and they function in the maintenance of the stability of plant cell membranes [55]. More than 200 different plant sterols have now been identified. Phytosterols usually exist in two different forms: one form is in a free state, and the other is covalently bound to ester or glycosidic bonds. Recent studies have found that plant sterols regulate cholesterol metabolism-related proteins to reduce circulating cholesterol 
levels [56]. It helps alleviate the risk of lipid metabolism disorders, cardiovascular disease, diabetes, and tumours [57-60]. In addition, the intervention of plant sterols also has certain advantages in increasing the abundance of intestinal flora, regulating faecal metabolites, and ameliorating NAFLD [61]. The most common phytosterols are $\beta$-sitosterol, campesterol, and stigmasterol.

In the past, many studies have reported the therapeutic effects of stigmasterol in antioxidative activities, anti-inflammatory activities, regulation of lipid metabolism, and antitumour activities. Studies have shown that stigmasterol improves rat brain damage caused by ischaemia-reperfusion by reducing oxidative stress and inflammation [62]. Stigmasterol relieves collagen-induced arthritis in rats by inhibiting proinflammatory cytokines [63]. In mice, stigmasterol significantly reduces total lipid, and TG and TC levels in the liver as well as liver histopathological levels, and it reduces intestinal bile acid levels and increases faecal lipid levels to treat NAFLD [64]. Stigmasterol activates ROS production and calcium overload, and it induces mitochondrial apoptosis and inhibits tumour cell migration and angiogenesis [65]. It has also been reported that stigmasterol inhibits the JAK/STAT signalling pathway and blocks the cell cycle to exhibit certain antitumour effects [66]. Stigmasterol has shown certain potential for the treatment of metabolic diseases, but there is no report that stigmasterol can treat metabolic diseases through $\operatorname{PPAR} \gamma$.

A safety test of WST-1 was performed in the current study, and we found that stigmasterol at less than $1000 \mu \mathrm{M}$ was not significantly toxic to HepG2 cells. We also used free fatty acids in a high-fat cell model treated with different doses of stigmasterol. The stigmasterol treatment had the following effects in WT cells: intracellular pigmented lipid droplets were deposited; the lipid content was significantly decreased; the TG content was significantly decreased; the PPAR $\gamma, \mathrm{CPT}-2$ and UCP-1 mRNA levels were significantly increased; and the PPAR $\gamma$ and UCP-1 protein levels were significantly increased. Subsequently, our group generated a HepG2 cell line in which PPAR $\gamma$ was knocked down. Stigmasterol treatment in these PPAR $\gamma^{--}$cells showed the following results: there was no significant change in the deposition of coloured lipid droplets; there was no significant difference in the lipid content; there was no significant difference in TG content; and the PPAR $\gamma$ and UCP-1 mRNA and protein levels did not significantly change.

In this study, the main active ingredients of the Renshen and Chaihu herb pair were discovered using a network database system. In vitro experiments demonstrated that stigmasterol enhances the lipid metabolism of HepG2 cells through the PPAR $\gamma$-UCP-1 signalling pathway. In recent years, an increasing number of studies have focused on the discovery of PPAR dual agonists and even pan-agonists for the treatment of NAFLD. This study further explored the effect of stigmasterol on PPAR $\alpha$ and PPAR $\delta$, demonstrating its potential mechanism in the treatment of NAFLD.

\section{Data Availability}

The dataset generated from this study can be requested from the corresponding author (Prof. Bing Wang) for scientific purposes.

\section{Disclosure}

Qiang Zhang and Li Zhang are the co-first authors.

\section{Conflicts of Interest}

The authors declare that they have no conflicts of interest.

\section{Acknowledgments}

This study was supported by the Research Fund of National Natural Science Foundation of China Youth Science Fund Project (Grant no. 81704006), Shanghai Traditional Chinese Medicine Inheritance and Technological Innovation Project (Grant no. ZYKC2019035), Shanghai General Hospital Integrated Traditional Chinese and Western Medicine Special Project (Grant no. ZHYY-ZXYJHZX-201910), National Natural Science Foundation of China General Project (Grant no. 81774061) and Three-Year Action Plan (2021-2023) of Shanghai Municipality for Further Accelerating the Inheritance, Innovation and Development of Traditional Chinese Medicine (Grant No. ZY(2021-2023)-0205-04).

\section{Supplementary Materials}

There are 2 supplementary tables in the submitted supplementary materials. Supplemental Table 1: GO enrichment analysis of the intersection target of the herb pair and NAFLD. Supplemental Table 2: KEGG pathway enrichment analysis of the intersection target of the herb pair and NAFLD. (Supplementary Materials)

\section{References}

[1] M. E. Rinella, "Nonalcoholic fatty liver disease," JAMA, vol. 313, no. 22, pp. 2263-2273, 2015.

[2] F. Zhou, J. Zhou, W. Wang et al., "Unexpected rapid increase in the burden of NAFLD in China from 2008 to 2018: a systematic review and meta-analysis," Hepatology, vol. 70, no. 4, pp. 1119-1133, 2019.

[3] S. H. Ibrahim, P. Hirsova, and G. J. Gores, "Non-alcoholic steatohepatitis pathogenesis: sublethal hepatocyte injury as a driver of liver inflammation," Gut, vol. 67, no. 5, pp. 963-972, 2018.

[4] S. L. Friedman, B. A. Neuschwander-Tetri, M. Rinella, and A. J. Sanyal, "Mechanisms of NAFLD development and therapeutic strategies," Nature Medicine, vol. 24, no. 7, pp. 908-922, 2018.

[5] N. Chalasani, Z. Younossi, J. E. Lavine et al., "The diagnosis and management of nonalcoholic fatty liver disease: practice guidance from the American Association for the Study of Liver Diseases," Hepatology, vol. 67, no. 1, pp. 328-357, 2018.

[6] European Association for the Study of the Liver (EASL), European Association for the Study of Diabetes (EASD) and European Association for the Study of Obesity (EASO), "EASL-EASD-EASO Clinical Practice Guidelines for the management of non-alcoholic fatty liver disease," Diabetologia, vol. 59, no. 6, pp. 1121-1140, 2016.

[7] K.-Q. Shi, Y.-C. Fan, W.-Y. Liu, L.-F. Li, Y.-P. Chen, and M.-H. Zheng, "Traditional Chinese medicines benefit to nonalcoholic fatty liver disease: a systematic review and meta- 
analysis," Molecular Biology Reports, vol. 39, no. 10, pp. 9715-9722, 2012.

[8] H. Yao, Y.-J. Qiao, Y.-L. Zhao et al., "Herbal medicines and nonalcoholic fatty liver disease," World Journal of Gastroenterology, vol. 22, no. 30, pp. 6890-6905, 2016.

[9] Q. Chen, T. Wang, and J. Li, "Effects of natural products on fructose-induced nonalcoholic fatty liver disease (NAFLD)," Nutrients, vol. 9, no. 2, 2017.

[10] B. Boezio, K. Audouze, and P. Ducrot, "Network-based approaches in pharmacology," Molecular Informatics, vol. 36, no. 10, 2017.

[11] S. Li and B. Zhang, "Traditional Chinese medicine network pharmacology: theory, methodology and application," Chinese Journal of Natural Medicines, vol. 11, no. 2, pp. 110-120, 2013.

[12] J. Ru, P. Li, J. Wang et al., "TCMSP: a database of systems pharmacology for drug discovery from herbal medicines," Journal of Cheminformatics, vol. 6, no. 1, p. 13, 2014.

[13] X. Xu, W. Zhang, C. Huang et al., "A novel chemometric method for the prediction of human oral bioavailability," International Journal of Molecular Sciences, vol. 13, no. 6, pp. 6964-6982, 2012.

[14] M. Hong, S. Li, and H. Y. Tan, "A network-based pharmacology study of the herb-induced liver injury potential of traditional hepatoprotective Chinese herbal medicines," Molecules, vol. 22, no. 4, 2017.

[15] A. Daina, O. Michielin, and V. Zoete, "SwissTargetPrediction: updated data and new features for efficient prediction of protein targets of small molecules," Nucleic Acids Research, vol. 47, no. W1, pp. W357-W364, 2019.

[16] J. Pinero, J. M. Ramirez-Anguita, and J. Sauch-Pitarch, "The DisGeNET knowledge platform for disease genomics: 2019 update," Nucleic Acids Research, vol. 48, no. D1, pp. D845-D855, 2020.

[17] D. Szklarczyk, J. H. Morris, H. Cook et al., "The STRING database in 2017: quality-controlled protein-protein association networks, made broadly accessible," Nucleic Acids Research, vol. 45, no. D1, pp. D362-D368, 2017.

[18] Y. Zhou, B. Zhou, L. Pache et al., "Metascape provides a biologistoriented resource for the analysis of systems-level datasets," Nature Communications, vol. 10, no. 1, p. 1523, 2019.

[19] F. Yang, X. Dong, X. Yin, W. Wang, L. You, and J. Ni, “Radix Bupleuri: a review of traditional uses, botany, phytochemistry, pharmacology, and toxicology," BioMed Research International, vol. 2017, Article ID 7597596, 2017.

[20] Y.-X. Wang, Q.-y. Liu, M. Zhang et al., "Polysaccharides from bupleurum induce immune reversal in late sepsis," Shock, vol. 49, no. 4, pp. 451-459, 2018.

[21] H. Jiang, L. Yang, A. Hou et al., "Botany, traditional uses, phytochemistry, analytical methods, processing, pharmacology and pharmacokinetics of Bupleuri Radix: a systematic review," Biomedicine \& Pharmacotherapy, vol. 131, Article ID 110679, 2020.

[22] P. Zhou, W. Xie, S. He et al., "Ginsenoside Rb1 as an antidiabetic agent and its underlying mechanism analysis," Cells, vol. 8, no. 3, 2019.

[23] G.-Y. Su, Z.-Y. Li, R. Wang et al., "Signaling pathways involved in p38-ERK and inflammatory factors mediated the anti-fibrosis effect of AD-2 on thioacetamide-induced liver injury in mice," Food \& Function, vol. 10, no. 7, pp. 3992-4000, 2019.

[24] K.-H. Lu, C.-Y. Weng, W.-C. Chen, and L.-Y. Sheen, "Ginseng essence, a medicinal and edible herbal formulation, ameliorates carbon tetrachloride-induced oxidative stress and liver injury in rats," Journal of Ginseng Research, vol. 41, no. 3, pp. 316-325, 2017.

[25] G. Musso, M. Cassader, E. Paschetta, and R. Gambino, "Bioactive lipid species and metabolic pathways in progression and resolution of nonalcoholic steatohepatitis," Gastroenterology, vol. 155, no. 2, pp. 282-302, 2018.

[26] K. Neumann, B. Schiller, and G. Tiegs, "NLRP3 inflammasome and IL-33: novel players in sterile liver inflammation," International Journal of Molecular Sciences, vol. 19, no. 9, 2018.

[27] M. J. Watt, P. M. Miotto, W. De Nardo, and M. K. Montgomery, "The liver as an endocrine organ-linking NAFLD and insulin resistance," Endocrine Reviews, vol. 40, no. 5, pp. 1367-1393, 2019.

[28] Z. Safari and P. Gérard, "The links between the gut microbiome and non-alcoholic fatty liver disease (NAFLD)," Cellular and Molecular Life Sciences, vol. 76, no. 8, pp. 1541-1558, 2019.

[29] S. Gaul, A. Leszczynska, F. Alegre et al., "Hepatocyte pyroptosis and release of inflammasome particles induce stellate cell activation and liver fibrosis," Journal of Hepatology, vol. 74, no. 1, pp. 156-167, 2021.

[30] G. A. Garinis, B. Fruci, A. Mazza et al., "Metformin versus dietary treatment in nonalcoholic hepatic steatosis: a randomized study," International Journal of Obesity, vol. 34, no. 8, pp. 1255-1264, 2010.

[31] D. M. Torres, F. J. Jones, J. C. Shaw, C. D. Williams, J. A. Ward, and S. A. Harrison, "Rosiglitazone versus rosiglitazone and metformin versus rosiglitazone and losartan in the treatment of nonalcoholic steatohepatitis in humans: a 12month randomized, prospective, open- label trial," Hepatology, vol. 54, no. 5, pp. 1631-1639, 2011.

[32] M. O. Rakoski, A. G. Singal, M. A. M. Rogers, and H. Conjeevaram, "Meta-analysis: insulin sensitizers for the treatment of non-alcoholic steatohepatitis," Alimentary Pharmacology \& Therapeutics, vol. 32, no. 10, pp. 1211-1221, 2010.

[33] M. S. Kuchay, S. Krishan, S. K. Mishra et al., "Effect of empagliflozin on liver fat in patients with type 2 diabetes and nonalcoholic fatty liver disease: a randomized controlled trial (E-LIFT trial)," Diabetes Care, vol. 41, no. 8, pp. 1801-1808, 2018.

[34] Z. M. Younossi, V. Ratziu, and R. Loomba, "Obeticholic acid for the treatment of non-alcoholic steatohepatitis: interim analysis from a multicentre, randomised, placebo-controlled phase 3 trial," Lancet, vol. 394, no. 10215, pp. 2184-2196, 2019.

[35] A. J. Sanyal, N. Chalasani, K. V. Kowdley et al., "Pioglitazone, vitamin E, or placebo for nonalcoholic steatohepatitis," New England Journal of Medicine, vol. 362, no. 18, pp. 1675-1685, 2010.

[36] X. Wei, C. Wang, S. Hao, H. Song, and L. Yang, "The therapeutic effect of berberine in the treatment of nonalcoholic fatty liver disease: a meta-analysis," Evidence-Based Complementary and Alternative Medicine, vol. 2016, Article ID 3593951, 9 pages, 2016.

[37] C. O. Zein, L. M. Yerian, P. Gogate et al., "Pentoxifylline improves nonalcoholic steatohepatitis: a randomized placebocontrolled trial," Hepatology, vol. 54, no. 5, pp. 1610-1619, 2011.

[38] S. Rahmani, S. Asgary, G. Askari et al., "Treatment of nonalcoholic fatty liver disease with curcumin: a randomized placebo-controlled trial," Phytotherapy Research, vol. 30, no. 9, pp. 1540-1548, 2016.

[39] M. Daneshi-Maskooni, S. A. Keshavarz, M. Qorbani et al., "Green cardamom supplementation improves serum irisin, 
glucose indices, and lipid profiles in overweight or obese non-alcoholic fatty liver disease patients: a double-blind randomized placebo-controlled clinical trial," BMC Complementary and Alternative Medicine, vol. 19, no. 1, p. 59, 2019.

[40] P. Lefebvre, G. Chinetti, and J. C. Fruchart, "Sorting out the roles of PPAR in energy metabolism and vascular homeostasis," Journal of Clinical Investigation, vol. 116, no. 3, pp. 571-580, 2006.

[41] T. Hashimoto, W. S. Cook, C. Qi, A. V. Yeldandi, J. K. Reddy, and M. S. Rao, "Defect in peroxisome proliferator-activated receptor $\alpha$-inducible fatty acid oxidation determines the severity of hepatic steatosis in response to fasting," Journal of Biological Chemistry, vol. 275, no. 37, pp. 28918-28928, 2000.

[42] C. Xu, L.-l. Wang, H.-y. Liu, X.-b. Zhou, Y.-l. Cao, and S. Li, "C $333 \mathrm{H}$, a novel PPARalpha/gamma dual agonist, has beneficial effects on insulin resistance and lipid metabolism1," Acta Pharmacologica Sinica, vol. 27, no. 2, pp. 223-228, 2006.

[43] Z. Dlamini, P. Ntlabati, Z. Mbita, and L. Shoba-Zikhali, "Pyruvate dehydrogenase kinase 4 (PDK4) could be involved in a regulatory role in apoptosis and a link between apoptosis and insulin resistance," Experimental and Molecular Pathology, vol. 98, no. 3, pp. 574-584, 2015.

[44] J. Li, Q. Huang, and X. Long, "CD147 reprograms fatty acid metabolism in hepatocellular carcinoma cells through Akt/ mTOR/SREBP1c and P38/PPAR $\alpha$ pathways," Journal of Hepatology, vol. 6, no. 63, pp. 1378-1389, 2015.

[45] L. M. Laiglesia, S. Lorente-Cebrián, and L. MartínezFernández, "Maresin 1 mitigates liver steatosis in ob/ob and diet-induced obese mice," International Journal of Obesity, vol. 3, no. 42, pp. 572-579, 2018.

[46] L. M. Sanderson, T. Degenhardt, A. Koppen et al., "Peroxisome proliferator-activated receptor $\beta / \delta(\operatorname{PPAR} \beta / \delta)$ but not PPAR $\alpha$ serves as a plasma free fatty acid sensor in liver," Molecular and Cellular Biology, vol. 29, no. 23, pp. 6257-6267, 2009.

[47] I. Hidalgo, N. Nájera, E. Meaney et al., "Effects of (-)-epicatechin on the time course of the expression of perilipins in a diet-induced model of nonalcoholic steatohepatitis," The Journal of Nutritional Biochemistry, vol. 77, no. 77, Article ID 108296, 2020.

[48] K. Gao, J. Zhang, and P. Gao, "Qishen granules exerts cardioprotective effects on rats with heart failure via regulating fatty acid and glucose metabolism," Chinese Medicine, vol. 21, no. 15, 2020.

[49] S. Demine, P. Renard, and T. Arnould, "Mitochondrial uncoupling: a key controller of biological processes in physiology and diseases," Cells, vol. 8, no. 8, 2019.

[50] M. Shi, X.-Y. Huang, X.-Y. Ren et al., "AIDA directly connects sympathetic innervation to adaptive thermogenesis by UCP1," Nature Cell Biology, vol. 23, no. 3, pp. 268-277, 2021.

[51] M. A. El, I. G. Fantus, and B. A. Ait, "Beneficial effects of alpha-lipoic acid on hypertension, visceral obesity, UCP-1 expression and oxidative stress in zucker diabetic fatty rats," Antioxidants (Basel), vol. 8, no. 12, 2019.

[52] G. Musso, M. Cassader, E. Paschetta, and R. Gambino, "Thiazolidinediones and advanced liver fibrosis in nonalcoholic steatohepatitis," JAMA Internal Medicine, vol. 177, no. 5, pp. 633-640, 2017.

[53] D. P. Kumar, R. Caffrey, J. Marioneaux et al., “The PPAR $\alpha / \gamma$ agonist saroglitazar improves insulin resistance and steatohepatitis in a diet induced animal model of nonalcoholic fatty liver disease," Scientific Reports, vol. 10, no. 1, p. 9330, 2020.
[54] S. Lefere, T. Puengel, and J. Hundertmark, "Differential effects of selective- and pan-PPAR agonists on experimental steatohepatitis and hepatic macrophages," Journal of Hepatology, vol. 73, no. 4, 2020 .

[55] R. A. Moreau, L. Nyström, B. D. Whitaker et al., "Phytosterols and their derivatives: structural diversity, distribution, metabolism, analysis, and health-promoting uses," Progress in Lipid Research, vol. 70, pp. 35-61, 2018.

[56] W.-S. He, H. Zhu, and Z.-Y. Chen, "Plant sterols: chemical and enzymatic structural modifications and effects on their cholesterol-lowering activity," Journal of Agricultural and Food Chemistry, vol. 66, no. 12, pp. 3047-3062, 2018.

[57] J. H. Dumolt and T. C. Rideout, "The lipid-lowering effects and associated mechanisms of dietary phytosterol supplementation," Current Pharmaceutical Design, vol. 23, no. 34, pp. 5077-5085, 2017.

[58] C. E. Cabral and M. Klein, "Phytosterols in the treatment of hypercholesterolemia and prevention of cardiovascular diseases," Arquivos Brasileiros de Cardiologia, vol. 109, no. 5, pp. $475-482,2017$.

[59] F. Gao, G. Wang, L. Wang, and N. Guo, "Phytosterol nutritional supplement improves pregnancy and neonatal complications of gestational diabetes mellitus in a doubleblind and placebo-controlled clinical study," Food \& Function, vol. 8, no. 1, pp. 424-428, 2017.

[60] B. d. C. Couder-García, N. J. Jacobo-Herrera, A. ZentellaDehesa, L. Rocha-Zavaleta, Z. Tavarez-Santamaría, and M. Martínez-Vázquez, "The phytosterol peniocerol inhibits cell proliferation and tumor growth in a colon cancer xenograft model," Frontiers in Oncology, vol. 9, p. 1341, 2019.

[61] L. Song, Y. Li, D. Qu et al., "The regulatory effects of phytosterol esters (PSEs) on gut flora and faecal metabolites in rats with NAFLD," Food \& Function, vol. 11, no. 1, pp. 977-991, 2020.

[62] Q. Liang, J. Yang, and J. He, "Stigmasterol alleviates cerebral ischemia/reperfusion injury by attenuating inflammation and improving antioxidant defenses in rats," Bioscience Reports, vol. 40, no. 4, 2020.

[63] M. Ahmad Khan, A. H. M. G. Sarwar, R. Rahat, R. S. Ahmed, and S. Umar, "Stigmasterol protects rats from collagen induced arthritis by inhibiting proinflammatory cytokines," International Immunopharmacology, vol. 85, Article ID 106642, 2020.

[64] S. Feng, Z. Dai, A. B. Liu et al., "Intake of stigmasterol and $\beta$-sitosterol alters lipid metabolism and alleviates NAFLD in mice fed a high-fat western-style diet," Biochimica et Biophysica Acta (BBA) - Molecular and Cell Biology of Lipids, vol. 1863, no. 10, pp. 1274-1284, 2018.

[65] H. Bae, G. Song, and W. Lim, "Stigmasterol causes ovarian cancer cell apoptosis by inducing endoplasmic reticulum and mitochondrial dysfunction," Pharmaceutics, vol. 12, no. 6, 2020.

[66] K. Li, D. Yuan, and R. Yan, "Stigmasterol exhibits potent antitumor effects in human gastric cancer cells mediated via inhibition of cell migration, cell cycle arrest, mitochondrial mediated apoptosis and inhibition of JAK/STAT signalling pathway," Journal of the Balkan Union of Oncology (J BUON), vol. 23, no. 5, pp. 1420-1425, 2018. 